\title{
HMM-Based Gene Annotation Methods
}

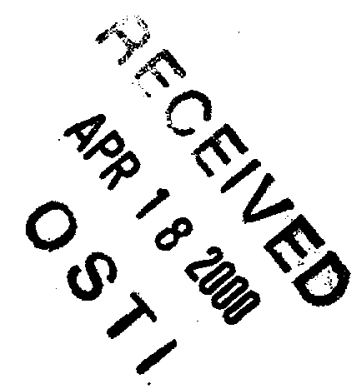

\section{Abstract}

We are developing new statistical methods and computational tools to identify genes in human genomic DNA, and to provide clues to their function by identifying features such as transcription factor binding sites, tissue-specific expression and splicing patterns, and remote homologies at the protein level with genes of known function. Our methods are based on extensions of the hidden Markov model (HMM) framework, which has been successful in numerous biosequence analysis applications. These extensions include the Fisher kernel method, a newly developed discriminative variant of HMM and related methods. Our focus in this research will be on extending our current methods to better identify features of the promoter region and to model alternative splicing, as well as improving our remote homology detection methods. We are members of the DOE Genome Annotation Consortium, and will incorporate our annotation tools into shared resources developed by this group, in addition to providing our own WWW server, to make these methods available to the widest possible user community. 


\section{DISCLAIMER}

This report was prepared as an account of work sponsored by an agency of the United States Government. Neither the United States Government nor any agency thereof, nor any of their employees, make any warranty, express or implied, or assumes any legal liability or responsibility for the accuracy, completeness, or usefulness of any information, apparatus, product, or process disclosed, or represents that its use would not infringe privately owned rights. Reference herein to any specific commercial product, process, or service by trade name, trademark, manufacturer, or otherwise does not necessarily constitute or imply its endorsement, recommendation, or favoring by the United States Government or any agency thereof. The views and opinions of authors expressed herein do not necessarily state or reflect those of the United States Government or any agency thereof. 


\section{DISCLAIMER}

Portions of this document may be illegible in electronic image products. Images are produced from the best available original document. 


\section{Contents}

1 Abstract 1

2 Aims $\quad 3$

3 Background and Results from Previous Support 3

3.1 Computational Genefinding . . . . . . . . . . . . . . . . . 3

3.2 Computational Protein Analysis $\ldots \ldots \ldots \ldots \ldots \ldots \ldots$

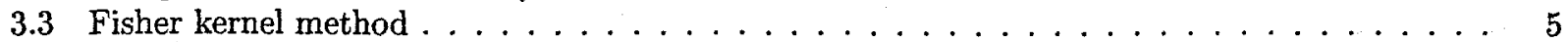

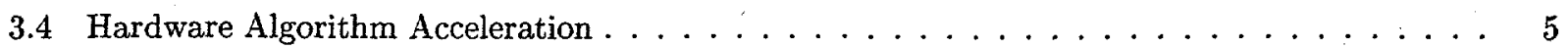

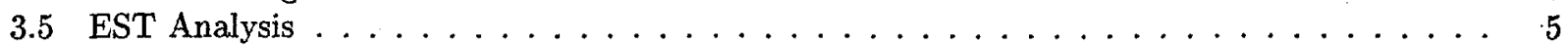

3.6 Stochastic Context Free Grammars and other RNA Research . . . . . . . . . . . . 6

4 Proposed Research 6

4.1 Using Multi-Spanning EST Derived Intron Evidence to Improve Gene Finding $\ldots \ldots$

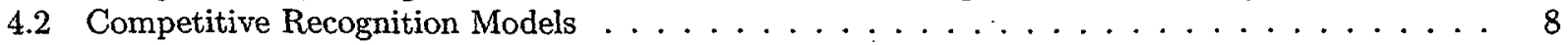

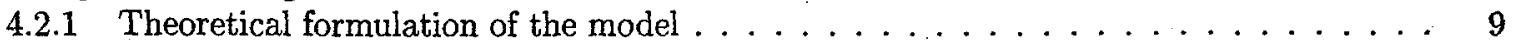

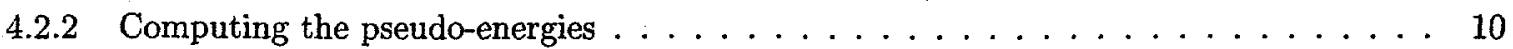

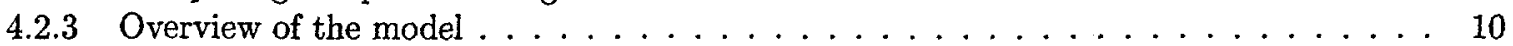

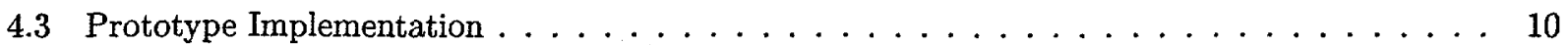

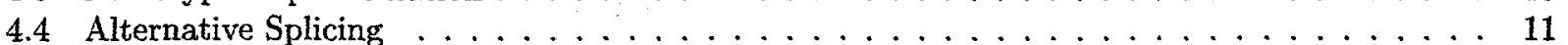

4.5 Gene Expression Analysis . . . . . . . . . . . . . . . . . . . . . . 12

4.6 GeneSpace . . . . . . . . . . . . . . . . . . . . . . . . . 13

4.7 Further work on Detecting Remote Protein Homologies . . . . . . . . . . . . . . . 14

4.8 EST Assembly and SNP detection . . . . . . . . . . . . . . . . . 15

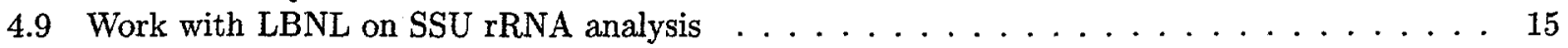

5 Conclusion $\quad 16$

6 Bibliography $\quad 16$

7 Biographical Sketches $\quad 24$

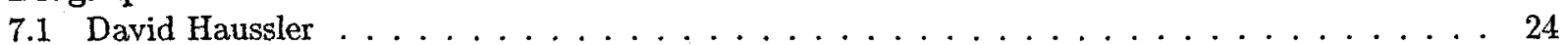

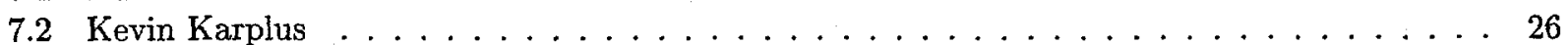

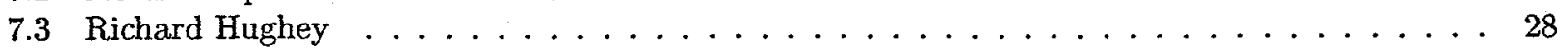

8 Budget Justification $\quad 30$ 


\section{Aims}

In the past four years we have developed a variety of methods for genome annotation and analysis based on hidden Markov models (HMMs). Our methods, and extensions of them, are now widely used by many laboratories. We have recently developed new methods that will significantly enhance and extend our previous work. The primary aim of this research is to complete the development and implementation of these new methods. The results will be

- better methods of incorporating EST, human-mouse synteny and other kinds of evidence into computational genefinding methods to improve their accuracy and enlarge the set of genomic features that they can handle,

- new computational gene structure models that represent and predict alternative splicing,

- integrated analysis of signals in upstream gene regions that control gene expression by combining sequence analysis with mRNA expression data,

- improved methods of detecting remote protein homologies using discriminative classification methods in conjunction with HMMs,

- improved methods for deriving consensus DNA and detecting SNPs from EST assemblies, and

- better computational methods for the multiple alignment and phylogenetic analysis of ribosomal RNA sequences.

The central theme of our proposed work is the computational integration of multiple kinds of evidence about genes obtained by high-throughput, genome-wide sequencing and functional genomics technologies. We propose statistical data models that will combine multiple types of such data to provide

- improved database annotation,

- interactive data exploration in a hypothesis-driven mode, and

- automated data mining in a data-driven mode.

We will provide internet access to all of the methods we develop, and incorporate our tools into the shared resources being developed by the DOE Genome Annotation Consortium, of which we are members.

\section{Background and Results from Previous Support}

Our previously supported work (DE-FG03-95ER62112, four years ending August 31, 1999), some of which was also funded in part by NSF, has resulted in 31 papers, most of which can be downloaded from our website $^{1}$. Below we discuss and cite these papers, along with related work of others. Graduate students Jim Alexander, Albion Baucom, Christian Barrett, Michael Brown, Melissa Cline, Jon Colburn, Mark Diekhans, Leslie Grate, Brad Gulko, Marc Hansen, Birong Hu, David Kulp, David Lin, Doanna Meads, Kimmen Sjölander, and Spencer Tu were supported or partially supported by this grant. These students include four women, who are underrepresented in computer science, as well as one underrepresented Hispanic student. In addition, during the DOE funding period the PIs have developed one new graduate course (CMPS243: bioinformatics ${ }^{2}$, and the groundwork has been laid for a UCSC Center for Biomolecular Engineering. ${ }^{3}$

\subsection{Computational Genefinding}

Computational methodology for finding genes and other functional sites in genomic DNA has evolved significantly over the last 20 years. Excellent recent surveys have been given by Gelfand [58], Fickett [54, 55], Guigó [75], Claverie [34], Milanesi and Rogosin [126], and Krogh [105] (see also [78]).

Through DOE funding, our laboratory pioneered the use of hidden Markov models (HMMs) [43] in genefinding. The parameters of a genefinding HMM can be estimated from a "training set" consisting of pieces of genomic DNA from a single organism annotated with the features one wishes to recognize, e.g. exons, introns, promoters, transcription factor binding sites, polyadenalation sites, LINEs, SINEs and other kinds of classified repetitive genomic DNA, begin and end of transcription, begin and end of translation, etc. The resulting statistical model can then be applied to predict the locations of these features in new sequences of genomic DNA from the same organism.

\footnotetext{
${ }^{1}$ http://ww .cse.ucsc.edu/research/compbio/research.html

${ }^{2}$ http://ww. cse.ucse.edu/classes/cmps243/Winter $98 /$

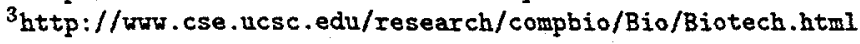


With Anders Krogh, we developed the first HMM genefinding program, called EcoParse, and applied it to $E$. coli [107]. This program was recently retrained and used in the annotation of the $M$. Tuberculosis genome [36]. Since that time, a number of other laboratories have developed HMM-based genefinding programs, making this a leading approach in the field. These include GeneMark-HMM (for bacterial genomes) [120] Veil [81] and HMMgene (for human) [105]. A somewhat more general class of probabilistic models, called generalized HMMs (GHMMs) or (hidden) semi-Markov models, have their roots in GeneParser [145], and were more fully developed in our genefinder Genie [111, 136, 110] and then GenScan [28] (see also [159]).

Under DOE support, we have developed special versions of Genie for finding C. elegans, D. melanogaster, and human/mouse genes $[111,110,136]$. In experimental tests, Genie and similar HMM-based genefinders mentioned above have proven to be the most effective computer programs for finding features in genomic DNA [105]. Genie has been extended to incorporate other kinds of experimental data into its predictions, in order to improve its accuracy. These other kinds of data include matches between pieces of the genomic DNA being analyzed and very similar sequences in the dbEST database of expressed sequence tags (ESTs) [158, 44, 94, 161] ("EST hits"), and matches in which the genomic DNA can be translated into a segment of protein that is very similar to a segment of a protein in a non-redundant protein database $[110,162]$ ("protein hits").

We have also developed a new method to incorporate protein hits into genefinding by using an HMM to represent a protein family, as discussed below, but making the "match" states of the model correspond to individual nucleic acids, rather than amino acids [109]. In this way, mutations, insertions, and deletions between the coding DNA and the protein family can be modeled at both the nucleotide and amino acid level. The resulting gene-finder is both robust to noise and sensitive to remote homologs. Related methods include the "spliced alignment" of Gelfand, Mironov, and Pevzner [60] and the "genewise" system developed by Ewan Birney [16, 15] Our approach is similar in spirit to [60], but we use an HMM for an entire protein family rather than a single homolog, our alignment strategy differs, and we combine homolog identification with additional statistical information provided by the gene structure GHMM. The approach is similar to Birney's approach in the use of the protein HMM, but we combine the protein HMM with a full gene structure GHMM, rather than a small, single-letter-per-state HMM of the classical kind.

In addition to the genomes discussed above, our HMM-based genefinding methods have recently been applied in conjunction with laboratory methods to obtain a more definitive annotation of introns in the yeast genome [147]. We are currently doing a similar study in $C$. elegans [65].

\subsection{Computational Protein Analysis}

With funding from DOE and NSF, our laboratory at UCSC pioneered the use of HMMs to detect remote homologies between protein domains, beginning with $[79,106]$ (see also the excellent related work in $[8,150$, $46,47,2,72]$, and see [43] for a complete introduction). The HMMs used in this research are different from those used in genefinding, and have a structure similar to that of a weight matrix or profile, $[69,68,21,82]$. HMMs for protein families have found several successful applications. They are used in the analysis of nematode and human DNA sequencing efforts at the genome center at Washington University and at the Sanger Centre [37]. The Pfam library of HMMs at the Sanger Centre now contains more than 1300 hidden Markov models for different protein families [146], a related library is being constructed at TIGR, and a large library has also been constructed at UCSC. ${ }^{4}$ HMMs led to the discovery of fibronectin type III domains in yeast [10] and members of the immunoglobulin superfamily in bacteria and bacteriophages [12, 13]. They were used in the analysis of lectins [80], ribonucleases [124], hedgehog proteins [40], endonucleases [39], helicases [153], leucine zipper motifs [57], steroid dehydrogenases [71], deaminase domains [125], and the Homocystinuria Disease Protein [11]. Recent comprehensive tests of HMM methods in identifying remote protein homologies in the SCOP database of protein structures [87] show that this method is significantly more powerful than methods such as BLAST [1] and FASTA [133] which are most commonly used in homology searching [132]. It also performed well at the two most recent blind tests of computational methods to predict protein structure (the CASP2 and CASP3 experiments, [99, 97]). In both cases, our group was among the top six in the fold recognition area that were chosen to speak and submit a paper to the special issue of Proteins: structure, function and genetics. We also competed successfully in a separate experiment in which only fully automatic procedures were permitted [101].

\footnotetext{
${ }^{4}$ http://ww .cse.ucsc.edu/research/compbio/HMM-apps/HMM-applications.html
} 
The Sequence Alignment and Modeling (SAM) software suite had its first general public distribution in $1995[90,88]$ and since then has been distributed to nearly 200 researchers. In our own studies $[9,95]$ as well as in external studies [123], SAM performance has been consistently better than standard methods and equal to or better than other HMM systems.

The HMM method we used in the most recent CASP experiment to identify remote protein homologies is called SAM-T98, and is available for general use at the above cited URL [98]. This web server allows remote homology searches to be performed without installing the SAM software. The user submits a protein sequence, and the SAM-T98 program builds an HMM model for that protein and its homologs (both domains and whole proteins) in the nonredundant protein database. These homologs are discovered automatically in the course of iterative model construction.

Additional research in protein analysis at UCSC has resulted in several further publications $[35,100,96$, $25,9,142,70,151,95]$.

\subsection{Fisher kernel method}

We have recently developed a new framework for detecting remote homologies among protein sequences that extends our previous HMM work [91, 92, 93]. The methodology, called the Fisher kernel method, exploits parametric models such as hidden Markov models in conjunction with powerful discriminant techniques that have greater potential to separate one type of sequence from another. In comparison with current HMM and BLAST methods, we demonstrate a nearly consistent, and in many cases substantial, increase in the accuracy of homology assessments for 33 SCOP families of structurally classified protein domains. These are all families with at least 5 structures in SCOP90, and at least ten structures for other domains in the same superfamily. They include G proteins, retroviral proteases, and interferons. others. Our tests use SCOP to simulate sequence-based recognition of remote homologies between novel protein sequences and those with known structural properties. While the present framework is developed primarily for assessing weak protein homologies it naturally extends to DNA parsing and other problems in biosequence analysis, which will be a focus of our proposed research.

\subsection{Hardware Algorithm Acceleration}

We have also developed a parallel processor called Kestrel that is a linear array processor originally tuned to sequence analysis applications including hidden Markov models [38, 85, 84, 90]. Kestrel's primary advantage is that it is generally programmable while keeping pace with single-purpose VLSI systems and while being vastly more cost-effective than general-purpose supercomputers [89]. The Kestrel processing element (PE) features an $8 \times 8$ multiplier, a $256 \times 8$ local memory, a $32 \times 83$-port shared register bank, a conditional and bit-processing shift register, and an ALU and minimizer.

With the help of NSF funding (PIs Hughey and Karplus), the Kestrel parallel processor is now a working system. The two single-board systems each have 512 processing elements, in 8 64-PE $0.5 \mu \mathrm{m}$ CMOS chips, and runs on a PC with a sockets interface to Unix machines. We have completed a rudimentary protein Smith \& Waterman alignment server using Kestrel, ${ }^{5}$ and, more importantly, have migrated HMM scoring, and will soon migrate HMM training to Kestrel. The Kestrel versions are over 20 times faster than those running on our DEC AlphaServer 4100, and the group is currently developing a greatly improved board that will provide an additional factor of three improvement. The Kestrel system will significantly reduce the time required for genome-wide HMM protein homology searches of a nonredundant database, model construction, and multiple alignment.

\subsection{EST Analysis}

With funding from SmithKline Beecham, we have developed a new algorithm, called ASA, for more accurate consensus inference in EST assembly. The algorithm identifies "noisy" regions of an EST assembly and applies a Markov Chain Monte Carlo (MCMC) technique to produced a maximum a posteriori probability (MAP) estimate of the consensus sequence for these regions $[114,115]$. In simulations on synthetic data with different coverage and error rates ranging from $5 \%$ to $30 \%$, ASA restored the consensus from noisy observations essentially as best as is theoretically possible for the given error rates. We also compared the

\footnotetext{
${ }^{5}$ http://www.cse.ucsc.edu/research/kestrel
} 
accuracy of ASA, CAP2, PHRAP and TIGR on ESTs from UNIGENE clusters with known mRNA. Our experiments showed that TIGR outperforms CAP2 and PHRAP, and in turn, ASA is slightly better than TIGR in terms of consensus accuracy, and uses significantly fewer wildcards.

We employ a neural network within the MCMC framework in ASA to model complex sequencing artifacts, such as gel compression. Using this model, ASA produces an optimal consensus as well as its possible alternatives with their relative probabilities in ambiguous regions. It can also provide a file with detailed reliability values that specify loss in log-probability when any consensus residue is modified using appropriate substitutions, insertions or deletion. When applied to EST assembly, this information on the accuracy of the consensus can be used further to correct possible frame-shifts and reconstruct ORFs. Finally, single nucleotide polymorphisms (SNPs) are explicitly incorporated in ASA's probabilistic model, and so ASA reports all SNPs it finds in the course of analysis and realignment as well.

\subsection{Stochastic Context Free Grammars and other RNA Research}

The pairwise interactions that form helices in RNA secondary structure are not well-modeled by HMMs, but we have shown that they can, with the exception of pseudoknots, be modeled adequately using stochastic context-free grammars (SCFGs), which constitute a class of stochastic models that includes HMMs as a special case $[137,138,139,64,152,66]$. Similar methods were developed independently by Sean Eddy and Richard Durbin [45, 119]; see also [116].

In collaboration with Harry Noller, director of the Center for Molecular Biology of RNA at UCSC, we have developed a general system for modeling RNA secondary structure using SCFG's This system provides tools for the specification of SCFG's for different RNA families, the estimation of model parameters given a set of training sequences, a scoring function that can be used to discriminate sequences which belong to the RNA family modeled from those that do not, and tools to produce multiple alignments of a set of sequences belonging to a RNA family. The system performs all of these tasks in a computationally efficient manner, making its application to very large RNA molecules feasible. Apart from our earlier experiments with tRNA [138] and snRNAs [152], the system's primary application has been the modeling of the small subunit of ribosomal RNA [22]. The size of this molecule is large: 1542 residues and 478 modeled basepair interactions. We have developed a web site that automatically determines the secondary structure of any bacterial small subunit ribosomal RNA ${ }^{6}$. In collaboration with Charles Wilson of the UCSC Biology Department we extended our system to employ intersections of SCFGs to model RNA pseudoknot interactions as well [23].

We have also experimented with Gibbs sampling $[64,66]$ and phylogenetic methods $[77,76]$ to determine the structure of the grammar itself from training sequences $[64,66]$. More recently, we have developed simplified methods to search for RNA patterns, to be used in conjunction with SCFGs to accelerate searches in large genomic databases. Using some of these methods, we recently discovered potential eukaryotic SECIS elements in the HIV-1 genome [67].

\section{Proposed Research}

\subsection{Using Multi-Spanning EST Derived Intron Evidence to Improve Gene Finding}

We plan to devote considerable attention to improving gene predictions using EST evidence. There are numerous anomalies in ESTs that make them difficult to use in genefinding, including base miscalls and indels, repeat sequences, incomplete mRNA splicing, and incorrect strand direction $[52,5]$

We have developed a prototype system that is capable of dealing with these issues, and deriving highly reliable implied introns from the noisy EST hit information. Initial results indicate that incorporating intron-evidence into the Genie gene finder can improve gene-finding performance by $5-12 \%$.

The system is based on finding multi-exon spanning ESTs, which are ESTs that include BLAST-recognizable hits to more than one exon in a gene structure. These were used in the analysis of $C$. elegans and in many other genefinding projects $[37,94,161]$. Multi-exon spanning ESTs are ESTs that match two or more regions of the genomic sequence separated by a distance sufficient to be an intron. We refer to the region that falls

\footnotetext{
${ }^{6}$ follow the link from http://wrk.cse.ucsc.edu/research/compbio
} 


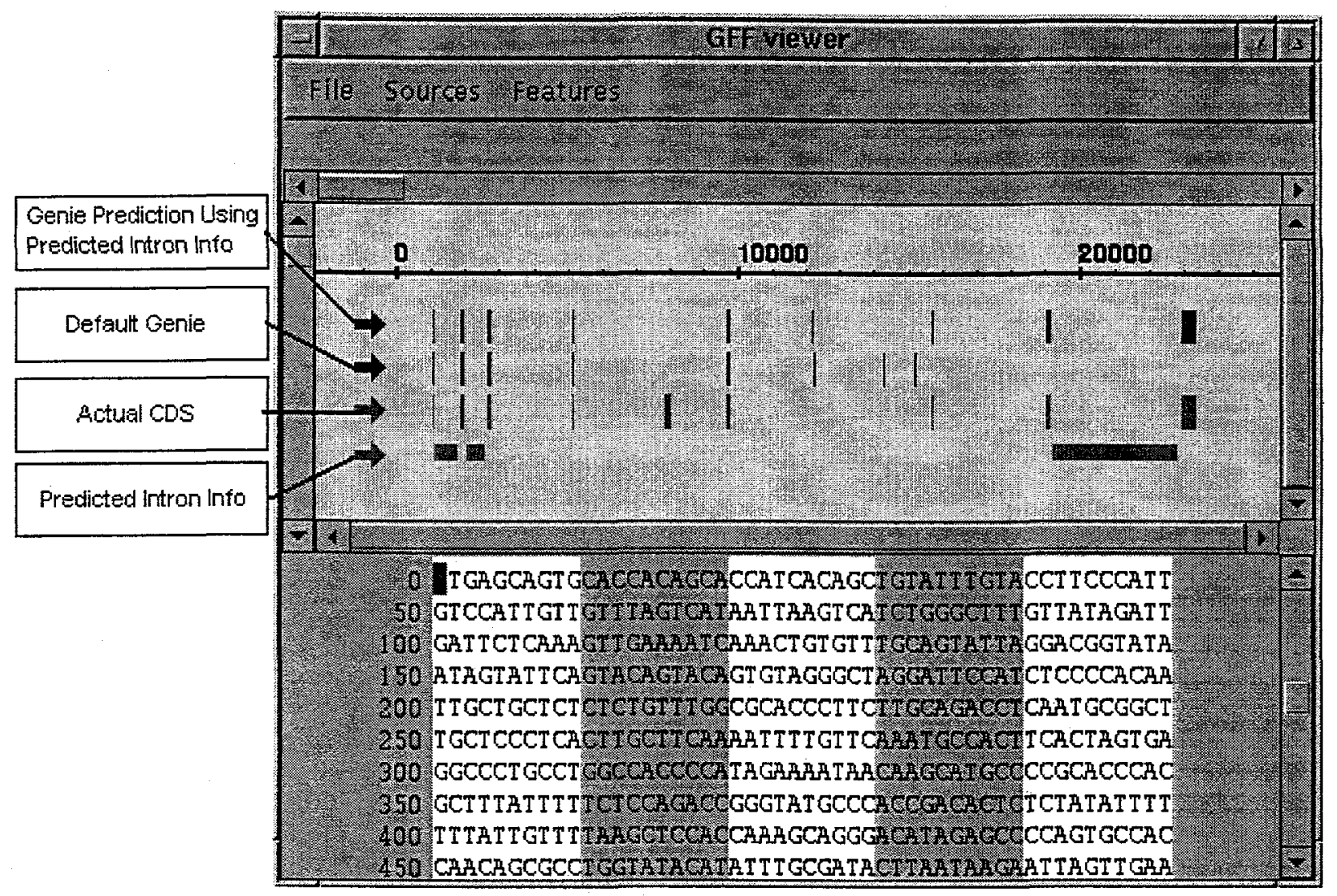

Figure 1: Comparison of Genie gene predictions with and without intron prediction evidence on an AT-rich gene(HUMIDS)

between the EST matches as an EST-intron. EST matches to repeat sequences are filtered out using RepeatMasker [143], and we use a score-based method to resolve discrepancies between predicted EST-introns and contradictory EST hits.

From our pilot test set of 64 human genes, this basic algorithm alone without any other genefinding tools, predicts 113 correct and 16 incorrect introns. Using a moderately stringent filter, the algorithm can predict 111 correct and 5 incorrect introns. Using the strictest parameter set, the program predicts 79 correct and no incorrect introns. When we add EST-introns as an additional sensor in Genie, we get a fully integrated genefinder that uses evidence from predicted EST-introns. Experiments show an improvement of $5 \%$ in predictive accuracy at both the single base and exact exon level for those genes with sufficient EST coverage (21 out of 64 genes). AT-rich genes, which currently present a great challenge for computational genefinding [30], show a greater improvement of $12 \%$.

This prototype program is still quite sensitive to errors in the predicted EST-introns because it does not use the full capability of Genie's probabilistic model. By scoring the individual bases in each predicted ESTintron using a likelihood ratio model, we should be able to improve the performance substantially. In such a model, bases incorrectly matched by BLAST at the ends of the predicted EST-introns would no longer have the strongly deleterious effects on gene prediction that they currently do, and neither would entirely incorrect EST-introns that are predicted on the basis of marginal evidence. In these cases, the likelihood ratios would indicate that these predictions were speculative, and Genie would incorporate them only if they increased the global probability of the predicted gene structure. Thus Genie would appropriately weigh the EST evidence against splice site, coding potential and other kinds of evidence currently built into to Genie's probabilistic model.

Our work on EST analysis $([114,115])$ has also shown the power of careful modeling of EST alignments. The whole is much greater than the sum of the parts when it comes to combining individual EST sequences. 
Performance on all genes with sufficient EST coverage

\begin{tabular}{|l|l|l|l|l|l|l|}
\hline & \multicolumn{2}{|c|}{ Base-level } & \multicolumn{4}{c|}{ Exon-level } \\
\hline & Sn & Sp & Sn & Sp & ME & WE \\
\hline Default Genie & 0.86 & 0.90 & 0.72 & 0.74 & 0.13 & 0.08 \\
\hline Genie with Intron & 0.93 & 0.92 & 0.78 & 0.77 & 0.07 & 0.08 \\
\hline
\end{tabular}

Performance on AT-rich genes only (AT Content> 55\%)

\begin{tabular}{|l|l|l|l|l|l|l|}
\hline & \multicolumn{2}{|c|}{ Base-level } & \multicolumn{4}{c|}{ Exon-level } \\
\hline & Sn & Sp & Sn & Sp & ME & WE \\
\hline Default Genie & 0.69 & 0.74 & 0.37 & 0.40 & 0.30 & 0.20 \\
\hline Genie with Intron & 0.88 & 0.77 & 0.52 & 0.47 & 0.17 & 0.27 \\
\hline
\end{tabular}

Base-level: Prediction accuracy per base; coding/non-coding

Exon-level: Accuracy with respect to exact prediction of exon start and end points

Sn: Sensitivity

Sp: Specificity

ME: Missing Exons: fraction of true exons that are not overlapped by any predicted.exon

WE: Wrong Exons: fraction of predicted exons that do not overlap any true exon

In the first 4 columns, higher values indicate better performance.

In the last 2 columis lower values indicate better performance.

By making EST assemblies on top of the genomic sequence, and doing a more careful analysis of these, we should be able to filter out many more EST anomalies than we current can using our prototype system. In particular, we will be able to eliminate more of the spurious EST matches that are due to repetitive DNA, many kinds of which are not currently masked by RepeatMasker. Also, by differentiating between ESTs from different libraries, we should be able to better handle incomplete mRNA splicing, and begin to distinguish this from true alternative splicing, as discussed further below.

\subsection{Competitive Recognition Models}

We use integrated probabilistic models for genefinding because existing local computational methods for detecting signals in nucleic acid sequences, such as weight matrices and neural nets, are of limited value when used in isolation. This is primarily because the short motifs that they are trying to recognize do not, by themselves, carry enough information to be reliably discriminated from similar-looking but nonfunctional regions in other parts of the genome. One might counter by arguing that since these sites are reliably distinguished in vivo by the protein or protein-RNA complex that binds to them, it is impossible that they do not carry enough information. However, this argument ignores the phenomena of cooperative and competitive binding. It is quite possible for a weak binding site to be used in preference to a nearby stronger binding site if by binding to the weak site, further interactions with neighboring bound proteins serve to form a stable complex, or if competition with some other configuration has rendered the stronger binding site inaccessible. Thus we would propose that computational methods that try to identify signals by any kind of local scoring scheme will, in principle, not be able to reach an extremely high accuracy.

Experience in constructing automated methods to determine gene structure confirms this claim. Nearly all successful gene-finding methods use some form of dynamic programming or depth-first search on a graph to combine the scores obtained from individual sensors into an optimal gene structure prediction. This global analysis of possible gene structures is essential in getting good performance. We propose an alternative global statistical model that is built from local models, but is itself non-local, and explicitly takes cooperation and competition into account. The key idea is that the statistical modeling of the interactions is done directly on the set of interpretations of a DNA sequence, rather than on the bases of the DNA sequence itself. Similar approaches have been used as alternatives to hidden Markov models in speech recognition (see e.g. $[19,20])$. The model is also related to the biosequence analysis models discussed in $[149,4,61]$, but it is not a segment-based model, and it uses a pseudo-energy formalism as in $[83,14]$, while combining this with a 
global Markov model in a new way. A closely related model has also recently been developed by Riis and Krogh [104].

\subsubsection{Theoretical formulation of the model}

Let $X=X_{1}, \ldots, X_{n}$ be a DNA sequence that consists of a gene and some surrounding untranscribed bases. Each $X_{i}$ represents a single base. An interpretation of $X$ is a sequence $Q=Q_{1}, \ldots, Q_{n}$, where each $Q_{i}$ is a state from a fixed set $\mathcal{S}$ of possible states. These states represent functional roles that may be assigned to a base in a particular context. For example, a very trivial set of states might be $\mathcal{S}=\left\{I, J, U, E_{1}, E_{2}, E_{3}\right\}$, where $E_{1}, E_{2}, E_{3}$ stand for positions 1 to 3 in a codon, $I$ stands for a base in an intron, $U$ stands for a base in an untranslated exon, and $J$ stands for a base in an intergenic region. In this case the interpretation

$$
Q=J J U U U E_{1} E_{2} E_{3} E_{1} I I I I I I I E_{2} E_{3} \ldots
$$

of the (toy) DNA sequence $X$ would assert that the first two bases of $X$ were not transcribed, that transcription begins at the third base, and the translated part of the gene begins at the sixth base, then after 4 bases the first exon ends and there is an intron of length seven, etc. This toy example will be used to illustrate the mathematical model behind competitive recognition models. In a realistic model, there would be many hundreds of states. Each position within every known signal would have a state associated with it. For example, there would be special states associated with the positions at and near 5' and 3' splice sites, branch points, states for positions within regulatory binding sites of different types, and states for introns within untranslated regions. If promoter and polyadenalation regions at either end of the gene are also modeled, there will be a very large number of states associated with functional features in these regions as well.

One can view the problem of annotating the features in a DNA sequence $X$ as the problem of finding the "correct" interpretation sequence $Q$ for it. However, in alternatively spliced genes, discussed further below, this view is clearly inappropriate. There is no single correct interpretation. Furthermore, even in genes that are not alternatively spliced, when a single interpretation is produced by computational analysis, there will inevitably be some uncertainty associated with it, and this uncertainty will be higher in some regions than in others. So here again, the notion of simply providing one interpretation is quite limiting. For these reasons, in competitive recognition models, we do not provide a single interpretation of a DNA sequence, but rather a probability distribution over all possible interpretations. Moreover, this probability distribution is parameterized, so that in different cellular environments, different interpretations become more likely. This is how we will represent alternative splicing.

Since the DNA sequence $X$ consists of $n$ bases, there are $n+1$ transition points in $X$, where we define a transition point to occur between every pair of consecutive bases, and also at the beginning and the end of $X$. Number the transition points of $X$ from 0 to $n$. Number the possible states in $\mathcal{S}$ from 1 to $M$. For each pair $i, j$ of possible states, and for each transition point $k$, define a pseudo-energy $H_{i j}(k)$. A pseudo-energy is a real number, and you can think of it as an energy that represents how favorable some local interpretation is. For example, using our toy example, suppose that state $i$ is $E_{2}$, the second position of a codon, and state $j$ is $I$, an intron state. Suppose that $k$ is a transition in the DNA sequence $X$ at a place that looks (locally) very much like a 5' splice site. It may look like a 5' splice site not only because it matches the consensus pattern for 5' splice sites well, but because hits from an EST database may align upstream of this position, and suddenly stop aligning at or near this point. In this case the pseudo-energy $H_{i j}(k)$ will be very low (perhaps even negative), indicating that this state transition is very favorable at this point in the DNA sequence. The pseudo-energies for the other possible state transitions at this point, $H_{i^{\prime} j^{\prime}}(k)$, will be much higher, indicating that these transitions are very unfavorable at this point in the sequence $X$.

Now consider a specific interpretation $Q=Q_{1}, \ldots, Q_{n}$ of $X$. At the transition point $k$, this interpretation asserts that a transition has been made from state $Q_{k}$ to state $Q_{k+1}$. The pseudo-energy of making this transition at this point in $X$ is $H_{Q_{k} Q_{k+1}}(k)$. We define the pseudo-energy of the entire interpretation $Q$ as the sum of the pseudo-energies of each of its transitions, i.e. $H(Q)=\sum_{k=0}^{n} H_{Q_{k} Q_{k+1}}(k)$ To make this precise, we define special states for the beginning and the end of the sequence $X$, and append these to the ends of the interpretation $Q$ as states $Q_{0}$ and $Q_{n+1}$, resp. Now, having defined the pseudo-energy of an interpretation, the probability of an interpretation can be defined by a Boltzmann-Gibbs distribution $P(Q)=e^{-H(Q)} / Z$ where the partition function $Z$ is the normalizing constant for this distribution, defined by $Z=\sum_{Q^{\prime}} e^{-H\left(Q^{\prime}\right)}$. Here the sum is over all possible interpretations $Q^{\prime}$. Of course this is a sum over 
exponentially many interpretations, but, as is well known, for this simple type of energy function, this sum can be computed efficiently using dynamic programming [19]. A similar dynamic programming algorithm can be used to find the single interpretation $Q$ with the highest probability. Thus this approach can be used to construct a single annotation of the sequence $X$ if one insists on the conventional view of the sequence annotation process. However, it is much richer than that. In particular, as discussed for a related model in $[145,149]$, this probability model can be used to answer questions such as "What is the probability that a 5' splice site occurs at position k?" or "What is the probability that the region from a to b is an exon?" [28]. Hence it represents the uncertainty in our predicted annotation as well. Again, the algorithms used to answer these questions use dynamic programming. The answers to all such questions are stored implicitly (and compactly) in the pseudo-energies $H_{i j}(k)$. So we argue that these pseudo-energies themselves represent the real annotation of the sequence $X$.

\subsubsection{Computing the pseudo-energies}

The key question that remains is how to obtain the pseudo-energies $H_{i j}(k)$ from the sequence $X$, and from auxiliary information such as aligned EST database hits, synteny with corresponding genomic regions of related organisms, and properties of the cell type from which we are analyzing this DNA. Here we propose to use a local discriminatively trained model such as a neural network or a kernel regression model. In particular, we will design a set of local discriminative models that look at a fixed size window of DNA, plus auxiliary information about that window, and output the pseudo-energies $H_{i j}(k)$ for all possible state transitions centered in that window.

A neural network is a parametric model; its weights are the parameters. By adjusting these weights, you can change the way the network computes the pseudo-energies. By providing the network with examples of correctly annotated DNA sequences, we can use gradient descent parameter estimation methods to train the neural net to produce pseudo-energies that assign the highest probability to the correct interpretation of the DNA sequence for a given tissue type or cellular state. In so doing, the neural net learns, indirectly, to annotate DNA. This also holds for other parametric models that can be used in place of neural nets for the purpose of estimating pseudo-energies, including kernel regression models. If we use local generative models, rather than local discriminative models, then competitive recognition models reduce to a special kind of graphical model [113], as described in [144, 108]. If these models are simple, then the competitive recognition model reduces to a simple HMM. Thus HMMs are a special case of competitive recognition models. The competitive recognition model approach allows one to build a more sophisticated annotation system on top of these simple methods.

\subsubsection{Overview of the model}

To summarize, competitive recognition models use local pattern recognition methods to determine a set of pseudo-energies for each transition point in a DNA sequence. Then the probabilistic modeling is done at the level of the interpretation of the DNA sequence, where each interpretation is viewed as a sequence of state transitions with assigned pseudo-energies. This is a much more powerful model than simply applying a local method with a fixed threshold. That would be like predicting each feature independently, simply by comparing its pseudo-energy to a fixed threshold. In competitive recognition models, the pseudo-energies are coupled to their neighbors. This coupling forms an inhomogeneous Markov chain structure defining a probability distribution over the set of all interpretations. Information from one part of the DNA sequence can be transmitted large distances by this Markov structure, so the overall model is highly non-local. In particular, in order to incorporate configurations of transitions with very low pseudo-energies in some parts of the interpretation, it will often be necessary to use transitions with higher pseudo-energies in other parts. In this way, the model can implicitly represent competitive and cooperative interactions among complexes that form on DNA, or on the corresponding pre-mRNA.

\subsection{Prototype Implementation}

In the last six months, we have begun a prototype implementation of a competitive recognition model for the annotation of genomic DNA. This system, called PGF, is designed to be scalable and flexible with respect to the gene features it is capable of modeling, the machine learning algorithms it employs, and the range of experimental data from which it learns. PGF is also designed to allow easy experimentation with various 
state models and state transition topologies. The system automatically transforms a high-level user-specified model into a detailed state transition model, adding frame information and transforming the topology to account for the user-specified length distributions of each feature. This modeling flexibility will allow the user to experimentally determine an appropriate level of model complexity.

In addition, PGF will be flexible with respect to the local discriminative models and machine learning algorithms it supports. The system is designed to incorporate arbitrary local models within a global, iterative learning scheme reminiscent of the expectation-maximization (EM) algorithm, but supports gradient descent as well as EM updates. The system is thus capable of learning using numerous variants of the standard hidden Markov model, including considerably simpler versions as well as more complex, context-sensitive competitive recognition models in which the local pseudo-energy depends an arbitrarily large context around the current position in the DNA sequence. PGF will also support neural network models and other discriminative models for computing pseudo-energies, including models that incorporate the Fisher kernel method [91, 92, 93].

Finally, in addition to its modeling and learning flexibility, PGF will be capable of learning from multiple types of experimental data. The user configures PGF with any number of parallel tracks, each of which holds a different type of experimental data about the bases in the genomic sequence being analyzed. These tracks can contain data derived from EST hits, similarities to known proteins, synteny between corresponding genomic regions in related organisms, or even gene predictions from human experts or other gene finding programs, which can provide important evidence for or against a hypothesized gene transcript. When more detailed experimental evidence is available, they can contain information about methylation of the bases, the probabilities that certain factors are bound to selected binding sites, and information about the topology of the DNA (bending, etc.) [7].

When training the local discriminative models, PGF presents to its core learning algorithm a view of the DNA sequence that includes a window of genomic DNA along with all the corresponding experimental data from the parallel tracks that is contained in that window. The learning algorithm then trains the model to take these additional data into account when computing pseudo-energies for the possible transitions at that position.

PGF is a carefully engineered software system designed to be usable for many years. It operates in an online fashion, so that it will be capable of continuously learning from the increasingly large quantities of molecular sequence data available. Furthermore, it is also being implemented on the Kestrel board, described above, for increased speed. In addition to allowing systematic experimentation, the system's flexibility will allow for future enhancements, reflecting our improving understanding of gene structure, the development of new machine learning algorithms, and the increasing availability of various types of experimental data.

After completing our implementation of PGF, we will first validate the system by applying it to the recently completed C. elegans genome, comparing its predictions to those made by Genie and other genefinders, and then retrain for mouse and human DNA. He we will use a discriminative training method based upon support vector machines as a core machine learning algorithm for the local functions that compute pseudo energies. A description of the complete project, including a detailed description of the algorithms involved, can be found at the PGF website?

\subsection{Alternative Splicing}

It has been estimated that $30 \%$ or more of human genes are alternatively spliced [59]. Most of these are spliced differently in different cell types, at different developmental stages, or in response to hormones and other external influences. Neural-specific alternative splicing appears to be especially prevalent [53,63, 148]. Alternatively spliced genes allow for regulation of protein activity and localization in the cell. Regulation of alternative splicing involves a complex arrangement of multiple splicing signals in the introns and exons of the pre-mRNA $[63,17,18]$. We are collaborating with Al Zahler in the Biology Department at UCSC to study a particular class of exonic sequences known as exonic splicing enhancers or ESEs that have been shown to both regulate alternative splicing and also to encode amino acids for translation (see [160] and references therein). These cis regulatory elements interact with proteins known as pre-mRNA splicing factors [103], which include SR proteins, an evolutionarily conserved family of proteins that can positively and negatively regulate splice site choice. The Zahler laboratory has shown that a subset of proteins belonging to the SR protein family has a strong direct binding interaction with an exonic splicing enhancer [129].

\footnotetext{
${ }^{7}$ http://www.cse.ucsc.edu/research/compbio/pgf
} 
Many signals in introns that participate in the regulation of alternative splicing have also been studied. In particular, several new factors have been identified that bind to intronic sequences in the neuron nucleus to regulate alternative splicing $[3,127,51,26,27,131]$. These proteins are related to several members of the heterogeneous nuclear ribonucleoprotein (hnRNP) family [42]. The importance of neuronal splicing regulation has recently been emphasized by the demonstration that in $70 \%$ of patients with amyotrophic lateral sclerosis (ALS or Lou Gehrig's disease), neurons in the motor cortex showed a strange pattern of alternative splicing of a glutamate transporter protein whereas neurons in other parts of the brain spliced this gene as expected [50]. That this disease is linked not to mutations in the glutamate transporter gene but to specific processing defects of the pre-mRNA shows the importance of alternative splicing in the analysis of the human genome.

Working with the Zahler laboratory, we will use the competitive recognition model framework described above to first annotate instances of alternatively spliced genes, and then construct models of alternative splicing. The key idea is that the pseudo-energy functions that determine the overall predicted gene structure can be trained to give the lowest energy to one splice variant under one set of conditions, and the lowest energy to an alternate splice variant under other conditions. This is possible because the pseudo-energy functions can depend on experimental or user-supplied information provided in parallel tracks along with the genomic DNA being analyzed, including EST information. In the simplest implementation, EST hits and user-supplied information about cell type can be used to directly train the pseudo-energy functions to produce the desired alternative splicing patterns in different cell types (see related work on detecting alternative splicing from ESTs in $[29,158,5])$. This will provide help with annotation of alternatively spliced genes in the short term. However, deeper models of alternative splicing can also be represented, and will become useful as more experimental data becomes available.

In these deeper models of alternative splicing, we include states in the competitive recognition model that model positions within key regulatory binding sites that control the splicing. Separate states are used to denote the regulatory site bound to the regulatory protein, and the regulatory site unbound. Then the local discriminative model that computes the pseudo-energies is trained so that in certain tissue types where the regulatory protein is absent or has very low concentration it assigns higher energy to transitions into the unbound state compared to transitions into the bound state in all positions where the regulatory site occurs. It is also trained to do the opposite in tissue types where the regulatory protein has higher concentration. Interactions modeled in the local pseudo-energies for positions in neighboring regions of the DNA can then be used to propagate this information to the relevant splice sites, which themselves will be represented by two kinds of states, corresponding to whether or not these splice sites are used. In this way, one set of splice sites will get high probability of being used in one tissue type, and alternate splice sites will have high probability in a different tissue type.

\subsection{Gene Expression Analysis}

Our other main area of research will be gene regulation, focusing on the annotation of transcription factor binding sites and other elements of the upstream regions of genes. To develop a more comprehensive gene expression analysis, and also to further explore alternative splicing experimentally, we have recently initiated a DNA microarray project at UCSC. The heart of this project is a robotic device used to spot DNA onto glass slides $[112,41]$. This "microarrayer" was developed by P. Brown and his associates, and we have constructed a similar device. While the microarrayer is initially being primarily used for yeast and C. elegans expression studies, a year from now we plan to start using it for mouse and human gene expression as well. Recent studies in yeast have shown how expression analysis, combined with MEME [6] computational exploration of sequence motifs in the promoter region, can lead to the automatic identification of transcription factor binding sites [33]. Working with our DOE-Sloan postdoc, Bill Grundy, who developed the meta-MEME system [72], we will be extending this work to build an automated system that finds potential transcription factor binding sites by maximizing likelihood in a mixture of meta-MEME models of the promoter region, where the components of the mixture are composed of sets of genes with closely related expression patterns over a large set of experimental conditions. As the data become available, this system will be adapted to the annotation of potential transcription factor binding sites in mouse and human genes.

These predicted transcription factor binding sites will be further studied in a computational manner by incorporating them into the state model of the PGF competitive recognition genefinding system described 
above. One immediate assessment of their value will be obtained by measuring how much they improve gene recognition on a benchmark test set of genes with known structure. This will give us one, albeit somewhat weak and indirect, method of validating our predictions. We plan to collaborate with Manny Ares and Grant Hartzog in the department of Biology at UCSC on direct experimental validation of the more interesting predictions.

Currently, the beginnings of genes are hard to recognize by computational methods because promoter regions have a highly variable and poorly understood structure $[86,32,135,122,134,56]$. There is considerable evidence that cooperative and competitive binding play a significant role in molecular recognition of promoter regions, and in the control of transcription [56, 163]. Thus there is reason to believe that the competitive recognition models we are building will be more accurate probabilistic models of these upstream regions. As our knowledge of promoter structure grows, we anticipate that these models will not only help with gene annotation, but will provide a quantitative simulation of gene regulation that biologists can use to explore different theoretical models of particular regulatory systems. They will provide an significant enhancement to current transcription factor databases [62, 24, 156, 102].

Once we have built general probabilistic model for the upstream regions of genes, either with meta-MEME or with PGF, we can also attempt to use this model to classify genes according to features of their promoter regions. Groundbreaking work has been done in this area by Jim Fickett and his colleagues [154] in which they took features from the promoter regions of muscle-specific human genes and used logistic regression to train a classifier that was able to distinguish many other muscle-specific genes from non-muscle-specific genes, and from regions of the genome that were not promoters at all. To make this research more generally applicable, an automated way to extract features from promoter regions for use in training such classifiers is needed. The Fisher kernel method, mentioned above and described more fully in [91, 92], provides just this. Using this method, we can extract the Fisher score parameters from either a meta-MEME or a PGF model of the promoter region, and use these directly with either logistic regression or support vector machines [141], to train a classifier that distinguishes different types of genes based on their promoter regions. In collaboration with Jim Fickett, we have already implemented this idea and are beginning to try it on his dataset.

With the availability of larger datasets of gene expression under different experimental conditions, the problem of classification of genes from their promoter regions will get considerably more attention. These datasets allow us to directly quantify how well a computational model is able to predict gene expression from genomic data. By providing large sets of train and test data for this problem, they will transform this field of research. We feel that our models can make a significant impact.

\subsection{GeneSpace}

It has been widely acknowledged that computational analysis can become a serious bottleneck in many aspects of large-scale gene expression studies $[48,140,118,155]$. As we move beyond the analysis of pure gene expression data to the exploration of relationships between expression patterns and other aspects of genes, including signals in upstream regions that may serve as transcription factor binding sites, signals in introns that may control alternative splicing, and signals involved in alternative 3 ' end formation, the computational approaches needed become significantly more complex. When you add in information about the gene from other types of functional genomics experiments, from properties of its protein product, and from homologs of the gene in other organisms, the bioinformatics challenges become severe. We plan to provide two types of bioinformatics tools to address these complex kinds of gene analysis:

1. A relational database that links tables of gene expression data, described by basic attributes of the gene (accession number, organism, etc.) and attributes of each gene expression experiment (primary expression data from the scanner for all genes, plus attributes of the experiment), with other tables that give features of the gene structure and the upstream and downstream regions from the gene, and further tables that give properties of the protein, homologs, functional predictions, etc. Scientists will be able to use SQL queries to these tables to gain basic access to any subset of the data they are interested in.

2. Statistical analysis of the data providing projections onto low dimensional subspaces (using projection pursuit and kernel PCA methods), analysis of correlations and clustering of the data (using standard Gaussian methods as in [48] and also non-linear kernel methods with K-means clustering [141], and 
predictive modeling from the data (using kernel-based regression and classification techniques that employ hidden variables; see below). These tools will provide answers to questions such as "What genes of unidentified function have expression patterns similar to these genes of known function?", "Knowing the expression pattern of a gene, how well can I predict certain features of its upstream region, and vice-versa?", and "Can the observed correlations between the expression patterns of several genes be statistically explained by a single hidden random variable representing an unobserved cause for these patterns?".

We intend these tools to be used not only in the semi-automated annotation of genomes, but also in both hypothesis-driven and data-driven investigations. Correlations, clusters, hidden explanatory variables, and other features of our genome-scale analysis tools will by themselves suggest scientific hypotheses which will be tested in the laboratory.

A uniform analysis of the data in the disparate tables described above is made possible by a uniform representation that we call GeneSpace. The key idea here is to extract real-valued features for all aspects of each gene, including aspects of the genomic DNA sequence and the protein sequence it codes for. This is possible using the Fisher kernel representation. The Fisher kernel allows us to apply hidden Markov and the more general kinds of probabilistic models discussed above to both genomic and protein sequences, and from these probabilistic models to extract real-valued features that capture essential functional elements in the sequences. Our experiments on remote homology detection between protein sequences show the power of this method [91]. By correlating these real-valued features with gene expression levels, we can explore the statistical interrelationships between features of the genomic DNA, features of the protein sequence, and the gene's expression levels under various experimental conditions. Using kernel-based classification methods, such as support vector machines [141], we can then form and evaluate predictive models from this data. We can ask, for example, how well certain features of the promoter region, combined with expression levels in certain experiments, and certain properties of the protein sequence made by the gene can, in combination, predict the functional classification of the gene. To do this, we begin with a set of genes with experimentally verified functions, divide it randomly into a train and test set (using possibly many different divisions), train a kernel-based classifier to predict the gene's function on the basis of the chosen features using the training set, and test its performance on the test set. If the prediction of gene function on the test set is successful with high probability, then not only have we shown that the features we chose were sufficient to predict gene function, but we have found a computational method of predicting gene function that can then be applied to other genes in the database of currently unknown function. The emphasis here is on the development of experimentally predictive models, rather than mere clustering of the data. Such predictive models represent the next logical step in gene expression analysis.

\subsection{Further work on Detecting Remote Protein Homologies}

We are encouraged by the initial results we have obtained by applying the Fisher kernel method to detect remote protein homologies [91]. Our experiments show that this method significantly improves on previous methods for the classification of isolated protein domains, as judged by SCOP superfamily definitions. In the next phase of this research, we will extend this methodology to deal with multidomain proteins. Here, rather than focusing on identifying exact domain boundaries, we will focus on identifying the exact location of key functional residues, such as active sites, or particular residues that are well conserved for structural reasons. The problem of identifying a particular active site residue can be set up as a classification problem, in a manner similar to that of classifying an entire domain. Hence it is amenable to the same kind of discriminative training methods. We are confident that Fisher kernel methods will be useful for this problem as well. If they are, then they could also be used to improve protein alignments, which they currently cannot do.

Certain technical details of the method also require attention. Our current approach employs a combination of scores derived from several underlying HMMs, in analogy with family pairwise search homology methods using BLAST, developed by Bill Grundy and others [73]. To date, we have only explored very simple methods for computing these combined scores. We plan to explore more sophisticated methods in the next set of experiments [117]. Also, the discriminative SVM-Fisher method relies on the presence of multiple training examples from the superfamily of interest, and works best when these training sequences are not the same as those used to estimate the parameters of the underlying HMM. This presents us with 
an allocation problem, i.e., which sequences should be used for estimating the parameters of the HMM and which ones left for the discriminative method. This issue becomes especially important in cases where there are relatively few known sequences and homologs in the superfamily of interest. A possible solution to this problem would be to employ generic protein models rather than those tuned to the particular family of interest. By generic models we mean HMMs constructed on the basis of statistical properties of short amino acid sequences that map on to structurally conserved regions in proteins [31]. Since the role of the HMM in our discriminative formalism is to provide features relevant for identifying structural relationships, the use of such generic models seems quite natural. It also will allow us to map all proteins into a common feature space, as needed by the GeneSpace concept described above.

\subsection{EST Assembly and SNP detection}

In our development of the ASA program for consensus estimation and SNP detection from EST assemblies (section 3.5), we restricted ourselves to consensus inference without trace data or PHRAP quality values. This allows the method to be applied to UNIGENE and other EST clusters/assemblies made from dbEST, which contain neither trace data nor quality values. However, information from trace data, and especially PHRAP reliability values, when available, can be very helpful in improving the accuracy of the consensus sequence. In future work we plan to incorporate this information in ASA's consensus estimation. One of the possible ways to do this is to convert reliability values reported by PHRAP into probabilities of substitutions, insertions, and deletions, used in our algorithm, which are currently computed by our neural net. Another approach would be to add the PHRAP values as inputs to the neural net, retraining it to exploit this additional information.

In addition to improving ASA's accuracy, we are especially interested in refining ASA for better SNP detection. Indeed, it is impossible to completely disentangle the problem of detecting SNPs in EST alignments from the problem of detecting and repairing misalignments and sequencing errors in EST assemblies: one cannot be solved without the other[74]. Since ASA uses MCMC methods to perform a more complete exploration of the space of local multiple EST alignments weighted by their posterior probability, it should be better able to distinguish true SNPS from misalignments and sequencing errors than conventional algorithms.

\subsection{Work with LBNL on SSU rRNA analysis}

We are collaborating with DOE Genome Annotation Consortium members Manfred Zorn and Sylvia Spengler, and LBNL scientist Frank Olken to further incorporate our method for aligning ribosomal RNA using Stochastic Context-Free Grammars (SCFG's), described above, into the Ribosomal Database Project (RDP) [121]. Alignment of ribosomal RNA is important because this is the most common molecule used for phylogenetic tree inference, which relies heavily on accurate multiple alignment [128]. Historically, rRNA was used by Carl Woese to relate all organisms and reconstruct the tree of life [157]. Recently, Norman Pace pointed to an opportunity for an environmental genome survey in which rRNA is gathered worldwide from a diverse set of environments to provide a sequence based snapshot of the microbial biodiversity [130]. This will stress the current methods used at RDP to the breaking point, since their multiple alignment procedures involve a combination of computer alignment with significant reliance on human fine tuning [49]. This has already led to a bottleneck, as evidenced by the large number of unaligned rRNA sequences in the current RDP dataset. Full analysis of widescale environmental biodiversity projects will not be possible without further automation.

We have done a pilot study on bacterial rRNA sequences which shows that SCFGs can give alignments that are superior to those produced by other techniques. We measured performance by the number of correctly identified base pairs in the secondary structure. Zuker's MFOLD program, a dynamic programming algorithm that uses thermodynamic parameters identified $34 \%$ of the correct basepairs when the optimal structures were analyzed. A commonly used sequence alignment program, ClustalW, achieved $92 \%$ accuracy. The SCFG had the best performance out of all tested programs, achieving $99 \%$ accuracy. The tests were done on bacterial small subunit ribosomal RNA sequences. We plan to extend this work to include models of other phylogenetically diverse groups such as Eukaryotes and Archaea. By implementing these methods on the NERSC machines at LBNL, we will create a platform for accurate rRNA multiple alignment that will be up to the computational task of Pace's biodiversity survey. 


\section{Conclusion}

Under our first four years of DOE support we have developed a large and active research group in genome analysis at UCSC, and made significant contributions to this field. We are entering a crucial phase now where we must expand beyond the pure sequence analysis tasks we have focused on during our initial years to incorporate new kinds of functional genomics data, including genome-wide mRNA expression data. The frameworks we will develop will incorporate such data, and other kinds of functional genomics data sets as they become available. By integrated analysis of these disparate data sets we hope to provide much more powerful genome annotation and analysis tools.

We are also entering a crucial phase in the development of the Center for Biomolecular Engineering at UCSC, which will foster interdisciplinary research and training in genomics, and significantly widen the participation at UCSC in genome research. These new collaborations, and the collaborations with our colleagues in the DOE Genome Annotation Consortium, will greatly stimulate our research, and significantly enhance our productivity in the next phase of this project.

\section{Bibliography}

[1] Stephen F. Altshul, Warren Gish, Webb Miller, Myers Eugene W., and Lipman David J. Basic local alignment search tool. $J M B, 215: 403-410,1990$.

[2] K. Asai, S. Hayamizu, and K. Onizuka. HMM with protein structure grammar. In Proceedings of the Hawaii International Conference on System Sciences, pages 783-791, Los Alamitos, CA, 1993. IEEE Computer Society Press.

[3] M. Ashiya and P. J. Grabowski. A neuron-specific splicing switch mediated by an array of pre-mrna repressor sites: Evidence of a regulatory role for the polypyrimidine tract binding protein and a brainspecific ptb counterpart. RNA, 3:996-1015, 1997.

[4] I. E. Auger and C. E. Lawrence. Algorithms for the optimal identification of segment neighborhoods. Bull. Math. Biol., 51:39-54, 1989.

[5] L.C. Bailey, D. Searls, and G.C. Overton. Analysis of est-driven gene annotation in human genomic sequence. Genome Research, 8:362-376, 1998.

[6] Timothy L. Bailey and Charles Elkan. The value of prior knowledge in discovering motifs with MEME. In ISMB-95, pages 21-29, Menlo Park, CA, July 1995. AAAI/MIT Press.

[7] P. Baldi, S. Brunak, Y. Chauvin, and A. Krogh. Naturally occuring neucleosome positioning signals in human exons and introns. JMB, 263(4):503-510, 1996.

[8] P. Baldi, Y. Chauvin, T. Hunkapillar, and M. McClure. Hidden Markov models of biological primary sequence information. PNAS, 91:1059-1063, 1994.

[9] Christian Barrett, Richard Hughey, and Kevin Karplus. Scoring hidden Markov models. CABIOS, 13(2):191-199, 1997.

[10] A. Bateman. Fibronectin type III domains in yeast detected by a hidden Markov model. Current Biology, 6(12):1544-1547, December 1996.

[11] A. Bateman. The structure of a domain common to archaebacteria and the homocystinuria disease protein. Trends in Biochemical Sciences, 22(1):12-13, January 1997.

[12] A. Bateman, S. Eddy, and C. Chothia. Members of the immunoglobulin superfamily in bacteria. Protein Sci., 5:1939-1941, 1996.

[13] A. Bateman, S. Eddy, and V. Mesyanzhinov. A member of the immunoglobulin superfamily in bacteriophage t4. Virus Genes, 14:163-165, 1997.

[14] O.G. Berg and P.H. von Hippel. Selection of DNA binding sites by regulatory proteins. J. Mol. Biol., 193:723-750, 1987.

[15] E. Birney and R. Durbin. Dynamite: a flexible code generating language for dynamic programming methods used in sequence comparison. In ismb97, pages 56-64, 1997.

[16] Ewan Birney, J. Thompson, and T. Gibson. PairWise and SearchWise: finding the optimal alignment is a simultaneous comparison of a protein profile against all DNA translation frames. $N A R, 24: 2730-2739$, 1996.

[17] D. L. Black. Finding splice sites within a wilderness of rna. RNA, 1:763-771, 1995. 
[18] D. L. Black. Splicing in the inner ear: A familiar tune, but what are the instruments? Neuron, 20:165-168, 1998.

[19] H. Bourlard and N. Morgan. Connectionist Speech Recognition, a hybrid approach. Kluwer, 1993.

[20] H. Bourlard, N. Morgan, and S. Renals. Neural nets and hidden markov models - review and generalizations. Speech Communication, 11(2-3):237-246, 1992.

[21] J. U. Bowie, R. Lüthy, and D. Eisenberg. A method to identify protein sequences that fold into a known three-dimensional structure. Science, 253:164-170, 1991.

[22] Michael Brown. Automatic ribosomal RNA alignment using threading by a porbabilistic SCFG/HMM. Poster Session First International Conference on RNA Structure, University of California at Santa Cruz, June 1997.

[23] Michael Brown and Charles Wilson. RNA pseudoknot modeling using intersections of stochastic context free grammars with applications to database search. In Pacific Symposium on Biocomputing, pages 109-125. World Scientific, 1995.

[24] P. Bucher. The eukaryotic promoter database, release 50. ftp://ftp.ebi.ac.uk/pub/databases/epd/, 1997.

[25] Philipp Bucher, Kevin Karplus, Nicolas Moeri, and Kay Hoffman. A flexible motif search technique based on generalized profiles. Computers and Chemistry, 20(1):3-24, January 1996.

[26] R. J. Buckanovich and R. B. Darnell. The neuronal rna binding protein nova-1 recognizes specific rna targets in vitro and in vivo. Molecular and Cellular Biology, 17:3194-3201, 1997.

[27] R. J. Buckanovich, Y. Y. Yang, and R. B. Darnell. The onconeural antigen nova-1 is a neuronspecific rna-binding protein, the activity of which is inhibited by paraneoplastic antibodies. Journal of Neuroscience, 16:1114-1122, 1996.

[28] C. Burge and S. Karlin. Predictions of complete gene structures in human genomic DNA. JMB, 268:78-94, 1997.

[29] J. Burke, H. Wang, W. Hide, and D. B. Davidson. Alternative gene form discovery and candidate gene selection from gene indexing projects. Genome Research, 8(3):276-290, 1998.

[30] M. Burset and R. Guigo. Evaluation of gene structure prediction programs. Genomics, 34(3):353367, 1996. Data set and evaluation results can be found at http://www.imim.es/GeneIdentification/Evaluation/Index.html.

[31] C. Bystroff and D. Baker. Blind predictions of local protein structure in casp2 targets using the i-sites library. Proteins: Structure, Function and Genetics, Suppl., 1:167-171, 1997.

[32] Q. K. Chen, G. Z. Hertz, and G. D. Stormo. MATRIX SEARCH 1.0: a computer program that scans DNA sequences for transcriptional elements using a database of weight matrices. CABIOS, 11(5):563-566, 1995.

[33] S. Chu, J. Derisi, M. Eisen, J. Mulholland, D. Botstein, P. Brown, and I. Herskowitz. The transcriptional program of sporulation in budding yeast. Science, 282:699-705, October 1998.

[34] J.-M. Claverie. Computational methods for the identification of genes in vertebrate genomic sequences. Human Molecular Genetics, 6(10):1735-1744, 1997.

[35] Melissa Cline and Kevin Karplus. On alignment shift and its measures. Technical Report UCSC-CRL97-27, University of California, Santa Cruz, Jack Baskin School of Engineering, UC Santa Cruz, CA 95064, February 1998.

[36] S. Cole et al. Deciphering the biology of mycobecterium tuberculosis from the complete genome sequence. Nature, 393(6685):537-544, 1998.

[37] Sequencing Consortium. Genome sequence of the nematode C. elegans: a platform for inv estigating biology. Science, 282:2012-2018, December 1998.

[38] David M. Dahle, Jeffrey D. Hirschberg, Kevin Karplus, Hansjörg Keller, Eric Rice, Don Speck, Douglas H. Williams, and Richard Hughey. Kestrel: Design of an 8-bit SIMD parallel processor. In Proc. 17th Conf. on Advanced Research in VLSI, pages 145-162. IEEE Computer Society, Sept 1997.

[39] J.Z. Dalgaard, A. Klar, M.J. Moser, A. Holley, W.R. Chatterjee, and I.S. Mian. Statistical modeling and analysis of the LAGLIDADG family of site-specific endonucleases and identification of an intein that encodes a site-specific endonuclease of the I-TevIII family. NAR, 25(22):4626-4638, 1997.

[40] J.Z. Dalgaard, M.M. Moser, R. Hughey, and I.S. Mian. Statistical modeling, phylogenetic analysis and structure prediction of a protein splicing domain common to inteins and hedgehog proteins. Jour. Comp. Biol., 4(2):193-214, 1997. 
[41] J.L. DeRisi, V.R. Iyer, and P.O. Brown. Exploring the metabolic and genetic control of gene expression on a genomic scale. Science, 278:680-686, 1997.

[42] G. Dreyfuss. hnrnp proteins and the biogenesis of mrna. Annual Review of Biochemistry, 62:289-321, 1997.

[43] R. Durbin, S. Eddy, A. Krogh, and G. Mitchison: Biological Sequence Analysis: Probabilistic Models of Proteins and Nucleic Acids. Cambridge University Press, 1998.

[44] B. A. Eckman, J. S. Aaronson, J. A. Borkowski, W. J. Bailey, K. O. Elliston, A. R. Williamson, and R. A. Blevins. The Merck Gene Index browser: an extensible data integration system for gene finding, genecharacterization and EST data mining. Bioinformatics, 14(1):2-13, 1998.

[45] S. R. Eddy and R. Durbin. RNA sequence analysis using covariance models. NAR, 22:2079-2088, 1994.

[46] Sean Eddy. Multiple alignment using hidden Markov models. In Christopher Rallings et al., editors, ISMB-95, pages 114-120, Menlo Park, CA, July 1995. AAAI/MIT Press.

[47] S.R. Eddy, G. Mitchison, and R. Durbin. Maximum discrimination hidden Markov models of sequence consensus. J. Comput. Biol., 2:9-23, 1995.

[48] M. Eisen, P. Spellman, P.O. Brown, and D. Botstein. Cluster analysis and display of genome-wide expression patterns. pnas, 95:14863-14868, December 1998.

[49] E.O'Brien, C.Notredame, and D. Higgins. Optimization of ribosomal rna profile alignemnts. Bioinformatics, 14(4):332-341, 1998.

[50] C. L. Lin et al. Aberrant rna prócessing in a neurodegenerative disease: The cause for absent eeat2, a glutamate transporter, in amyotrophic lateral sclerosis. Neuron, 20:589-602, 1998.

[51] H. Min et al. A new regulatory protein, ksrp, mediates exon inclusion through an intronic splicing enhancer. Genes and Development, 11:1023-1036, 1997.

[52] L. Hillier et al. Generation and analysis of 280,000 human expressed sequence tags. Genome Research, $5: 807-828,1996$.

[53] S. Stamm et al. A sequence compilation and comparison of exons that are alternatively spliced in neurons. Nucleic Acids Research, pages 1515-1526, 1994.

[54] J.W. Fickett. Finding genes by computer - the state of the art. Trends in Genetics, 12(8):316-320, 1996.

[55] J.W. Fickett. The gene identification problem - an overview for developers. Computers and Chemistry, 20(1):103-118, 1996.

[56] J.W. Fickett and A. G. Hatzigeorgiou. Eukaryotic promoter recognition. Genome Research, 7(9):861$878,1997$.

[57] Y. Fujiwara, M. Asogawa, and A. Konagaya. Hidden markov model to extract leucine zipper motif. NEC Research and Developments, 38:359-366, 1997.

[58] M. S. Gelfand. Prediction of function in DNA sequence analysis. Jour. Comp. Biol., 2(1):87-115, 1995.

[59] M. S. Gelfand, I. Dubchak, I. Dralyuk, and M. Zorn. Asdb: a database of alternatively spliced genes. NAR, 27(1):301-302, 1999.

[60] M. S. Gelfand, A. A. Mironov, and P. A. Pevzner. Gene recognition via spliced sequence alignment. PNAS, 93(17):9061-9066, 1996.

[61] M. S. Gelfand and M. A. Roytberg. Prediction of the exon-intron structure by a dynamic programming approach. BioSystems, 30:173-182, 1993.

[62] D. Ghosh. Tfd: The transcription factor database. NAR, 20S:2091-2093, 1992.

[63] P. J. Grabowski. Splicing regulation in neurons: tinkering with cell-specific control. Cell, 92:709-712, 1998.

[64] L. Grate, M. Herbster, R. Hughey, I.S. Mian, H. Noller, and D. Haussler. RNA modeling using Gibbs sampling and stochastic context free grammars. In ISMB-94, Menlo Park, CA, August 1994. AAAI/MIT Press.

[65] L. Grate, A. Jagota, B. Lazareva, T. Jaakkola, B. Martin, and D. Haussler. Dependencies and periodicities in C. elegans introns, 1999. in preparation.

[66] Leslie Grate. Automatic RNA secondary structure determination with stochastic context-free grammars. In Christopher Rallings et al., editors, ISMB-95, pages 136-144, Menlo Park, CA, July 1995. AAAI/MIT Press. 
[67] Leslie Grate. Potential SECIS elements in HIV-1 strain HXB2. Journal of AIDS and Human Retrovirology, 17(5):398-403, Apr 151998.

[68] M. Gribskov, R. Lüthy, and D. Eisenberg. Profile analysis. Methods in Enzymology, 183:146-159, 1990.

[69] Michael Gribskov, Andrew D. McLachlan, and David Eisenberg. Profile analysis: Detection of distantly related proteins. PNAS, 84:4355-4358, July 1987.

[70] J. A. Grice, R. Hughey, and D. Speck. Reduced space sequence alignment. CABIOS, 13(1):45-53, February 1997.

[71] W. N. Grundy, W. Bailey, T. Elkan, and C. Baker. Hidden markov model analysis of motifs in steroid dehydrogenases and their homologs. Biochemical and Biophysical Research Communications, 231:760-766, 1997.

[72] W. N. Grundy, W. Bailey, T. Elkan, and C. Baker. Meta-MEME: Motif-based hidden Markov models of protein families. CABIOS, 13(4):397-406, 1997.

[73] Willian N. Grundy. Family-based homology detection via pairwise sequence comparison. In Int. Conf. Computational Molecular Biology (RECOMB-98), New York, 1998. ACM Press.

[74] Z. Gu, L. Hillier, and P. Kwok. Single nucleotide polymorphism hunting in cyberspace. Human Mutation, 12:221-225, 1999.

[75] R. Guigo. Computational gene identification: an open problem. Computers and Chemistry, 21(4):215$222,1997$.

[76] B. Gulko and D. Haussler. Using multiple alignments and phylogenetic trees to detect rna secondary structure. In Proceedings of the Pacific Symposium on Biocomputing, pages 350-367. World Scientific Press., 1996.

[77] Brad Gulko. Using phylogenetic Markov trees to detect conserved structure in RNA multiple alignments. Master's thesis, University of California, Computer Science, UC Santa Cruz, CA 95064, 1995.

[78] D. Haussler. Computational genefinding. Trends Guide to Bioinformatics, supplement:12-14, 1998.

[79] D. Haussler, A. Krogh, I. S. Mian, and K. Sjölander. Protein modeling using hidden Markov models: Analysis of globins. In Proceedings of the Hawaii International Conference on System Sciences, volume 1, pages 792-802, Los Alamitos, CA, 1993. IEEE Computer Society Press.

[80] B. Hazes. The (QxW)3 domain: A flexible lectin scaffold. Protein Sci., 5(8):1490-1501, August 1996.

[81] J. Henderson, S. Salzberg, and K. Fasman. Finding genes in human DNA with a hidden Markov model. Journal of Computational Biology, 4(2):119-126, 1997.

[82] Steven Henikoff and Jorja G. Henikoff. Automated assembly of protein blocks for database searching. NAR, 19(23):6565-6572, 1991.

[83] J. Heumann, A. Lapedes, and G. Stormo. Neural networks for determining protein specificity and multiple alignment of binding sites. In Altman et al., editor, ISMB-94, pages 188-194. AAAI press, 1994.

[84] J. D. Hirschberg, D. Dahle, K. Karplus, D. Speck, and R. Hughey. Kestrel: A programmable array for sequence analysis. Journal of VLSI Signal Processing, 19:115-126, 1998.

[85] Jeffrey D. Hirschberg, Richard Hughey, Kevin Karplus, and Don Speck. Kestrel: A programmable array for sequence analysis. In Application-Specific Array Processors, pages 25-34, Los Alamitos, CA, July 1996. IEEE Computer Society.

[86] P. B. Horton and M. Kanehisa. An assessment of neural network and statistical approaches for prediction of e.coli. promoter sites. Nucleic Acids Research, 20:4331-4338, 1992.

[87] T. Hubbard, A. Murzin, S. Brenner, and C. Chothia. SCOP: a structural classification of proteins database. NAR, 25(1):236-9, January 1997.

[88] R. Hughey and A. Krogh. SAM: Sequence alignment and modeling software system. Technical Report UCSC-CRL-95-7, University of California, Santa Cruz, Computer Engineering, UC Santa Cruz, CA 95064, 1995.

[89] Richard Hughey. Parallel sequence comparison and alignment. CABIOS, 12(6):473-479, 1996.

[90] Richard Hughey and Anders Krogh. Hidden Markov models for sequence analysis: Extension and analysis of the basic method. CABIOS, 12(2):95-107, 1996. Information on obtaining SAM is available at http://www.cse.ucsc.edu/research/compbio/sam.html.

[91] T. Jaakkola, M. Diekhans, and D. Haussler. A discriminative framework for detecting remote protein homologies, 1998. Unpublished, available from http://www.cse.ucsc.edu/research/compbio/ research.html. 
[92] T. Jaakkola and D. Haussler. Exploiting generative models in discriminative classifiers. In Advances in Neural Information Processing Systems 11, San Mateo, CA, 1998. Morgan Kauffmann Publishers. to appear.

[93] T. Jaakkola and D. Haussler. Probabilistic kernel regression models. In Proc. of the Seventh Int. Workshop on $A I$ and Statistics, 1998. to appear.

[94] J. Jiang and H. J. Jacob. EbEST: an automatic tool using expressed sequence tags to delineate gene structure. Genome Research, 8(3):268-275, 1998.

[95] Rachel Karchin and Richard Hughey. Weighting hidden Markov models for maximum discrimination. Bioinformatics to appear, 1998.

[96] Kevin Karplus. Regularizers for estimating distributions of amino acids from small samples. In ISMB95, Menlo Park, CA, July 1995. AAAI/MIT Press.

[97] Kevin Karplus, Christian Barrett, Melissa Cline, Mark Diekhans, Leslie Grate, and Richard Hughey. Predicting protein structure using only sequence information. Proteins: Structure, Function, and Genetics, to appear, 1999.

[98] Kevin Karplus, Christian Barrett, and Richard Hughey. Hidden markov models for detecting remote protein homologies. Bioinformatics, 14(10):846-856, 1998.

[99] Kevin Karplus, Kimmen Sjölander, Christian Barrett, Melissa Cline, David Haussler, Richard Hughey, Liisa Holm, and Chris Sander. Predicting protein structure using hidden Markov models. Proteins: Structure, Function, and Genetics, Suppl. 1:134-139, 1997.

[100] Kevin Karplus, Kimmen Sjölander, Christian Barrett, Melissa Cline, David Haussler, Richard Hughey, Liisa Holm, and Chris Sander. Predicting protein structure using hidden Markov models, the CASP2 contest. Technical Report UCSC-CRL-97-13, University of California, Santa Cruz, Computer Science, UC Santa Cruz, CA 95064, November 1997.

[101] Lawrence A. Kelley, Robert M. MacCallum, Michael Sternberg, Kevin Karplus, Daniel Fischer, Arne Elofsson, Adam Godzik, Leszek Rychlewski, Krzysztof Pawłowski(A), David Jones, Kevin Bryson, and Burkhard Rost. CAFASP-1: Critical assessment of fully automated structure prediction methods. Proteins: Structure, Function, and Genetics, to appear, 1999.

[102] N. Kolchanov et al. GenExpress: a computer system for description, analysis, and recognition of regulatory sequences in eukaryotic genome. In Proceedings of the Fifth International Conference on Intelligent Systems for Molecular Biology, pages 95-104, 1998.

[103] A. Kramer. The structure and function of proteins involved in mammalian pre-mrna splicing. Annual Review of Biochemistry, 65:367-409, 1996.

[104] A. Krogh. Private communication.

[105] A. Krogh. Gene finding: putting the parts together. In Martin J. Bishop, editor, Guide to Human Genome Computing, chapter 11, pages 261-274. Academic Press, 2nd edition, 1998.

[106] A. Krogh, M. Brown, I. S. Mian, K. Sjölander, and D. Haussler. Hidden Markov models in computational biology: Applications to protein modeling. JMB, 235:1501-1531, February 1994.

[107] A. Krogh, I. S. Mian, and D. Haussler. A Hidden Markov Model that finds genes in E. coli DNA. NAR, 22:4768-4778, 1994.

[108] A. Krogh and S. Riis. Hidden neural networks. Neural Computation, 1998. too appear.

[109] D. Kulp and D. Haussler. Embedding HMMs: A Method for Recognizing Protein Homologs in DNA, 1997. http://www.ornl.gov/hgmis/publicat/97santa/infortoc.html.

[110] D. Kulp, D. Haussler, M. G. Reese, and F. H. Eeckman. Integrating database homology in a probabilistic gene structure model. In R. B. Altman, A. K. Dunker, L. Hunter, and T. E. Klein, editors, Proceedings of the Pacific Symposium on Biocomputing, pages 232-244. World Scientific, New York, 1997.

[111] D. Kulp, D. Haussler, M.G. Reese, and F. Eeckman. A generalized hidden Markov model for the recognition of human genes in DNA. In ISMB-96, pages 134-142, St. Louis, June 1996. AAAI Press. http://www.cse.ucsc.edu/ dkulp/cgi-bin/genie.

[112] D.A. Lashkari, J.L. DeRisi, J.H. McCusker, A.F. Namath, C. Gentile, S.Y. Hwang, P.O. Brown, and R.W. Davis. Yeast microarrays for genome wide parallel genetic and gene expression analysis. pnas, 94:13057-13062, 1997.

[113] S. Lauritzen. Graphical Models. Oxford University Press, 1996. 
[114] B. Lazareva and D. Haussler. A probabilistic approach to consensus multiple alignment. In R. B. Altman, A. K. Dunker, L. Hunter, and T. E. Klein, editors, Proceedings of the Pacific Symposium on Biocomputing, pages 150-161. World Scientific, New York, 1999.

[115] B. Lazareva and D. Haussler. Towards an accurate est consensus, 1999 . manuscript, see http://www.cse.ucsc.edu/ haussler/pubs.html.

[116] F. Lefebvre. A grammar-based unification of several alignment and folding algorithms. In ISMB-96, St. Louis, June 1996. AAAI Press.

[117] D. V. Lindley. Reconciliation of discrete probability distributions. Bayesian Statistics, 2:375-390, 1985.

[118] D. Lockhart et al. Expression monitoring by hybridization to high-density oligonucleotide arrays. Nature Biotechnology, 14:1675-1680, December 1996.

[119] T.M. Lowe and S.R. Eddy. tRNAscan-SE: a program for improved detection of transfer RNA genes in genomic sequence. NAR, 25:955-964, 1997.

[120] A. V. Lukashin and M. Borodovsky. Genemark.hmm: new solutions for gene finding. Nucleic Acids Research, 26(4):1107-1115, 1998.

[121] B. Maidak, G. Olsen, N. Larsen, R. Overbeek, M. McCaughey, and C.Woese. The ribosomal data project (rdp). NAR, 25:109-111, 1997.

[122] S. Matis, Y. Xu, M. B. Shah, D. Buley, X. Guan, J. R. Einstein, R. J. Mural, and E. C. Uberbacher. Detection of RNA Polymerase II Promoters and Polyadenylation Sites in Human DNA Sequence. Computers and Chemistry, 20:135-140, 1995.

[123] Marcella McClure, Chris Smith, and Pete Elton. Parameterization studies for the SAM and HMMER methods of hidden Markov model generation. In ISMB-96, pages 155-164, St. Louis, June 1996. AAAI Press.

[124] I.S. Mian. Sequence analysis of ribonucleases HII, III, II, PH and D. NAR, 25:3187-3195, 1997.

[125] I.S. Mian, M.J. Moser, W.R. Holley, and A. Chatterjee. Statistical modelling and phylogenetic analysis of a deaminase domain. Journal of Computational Biology, 5:57-72, 1997.

[126] L. Milanesi and I. Rogozin. Prediction of human gene structure. In Martin J. Bishop, editor, Guide to Human Genome Computing. Academic Press, 2nd edition, 1998.

[127] H. Min, R. C. Chan, and D. L. Black. The generally expressed hnrnp $f$ is involved in a neural-specific pre-mrna splicing event. Genes and Development, 9:2659-2671, 1995.

[128] D. Morrison and J. Ellis. Effects of nucleotide sequence alignment on phylogeny estimation: a case study of 18s rdnas of apicomplexa. Mol. Biol. Evol, 14:428-441, 1997.

[129] R. Nagel, A. Lancaster, and A. Zahler. Specific Binding of an Exonic Splicing Enhancer by the PremRNA Splicing Factor SRp55. RNA, 4:11-23, 1998.

[130] N.Pace. A molecular view of microbial diversity and the biosphere. science, 276:734-740, 1997.

[131] H. J. Okano and R. B. Darnell. A hierarchy of hu rna binding proteins in developing and adult neurons. Journal of Neuroscience, 17:3024-3037, 1997.

[132] J. Park, K. Karplus, C. Barrett, R. Hughey, D. Haussler, T. Hubbard, and C. Chothia. Sequence comparisons using multiple sequences detect twice as many remote homologues as pairwise methods. $J M B, 284(4): 1201-1210,1998$. Paper available at http://www.mrc-lmb.cam.ac.uk/genomes/jong/ assess_paper/assess_paperNov.html.

[133] W. Pearson and D. Lipman. Improved tools for biological sequence comparison. PNAS, 85:2444-2448, 1988.

[134] A. Pedersen, P. Baldi, S. Brunak, and Y. Chauvan. Characterization of prokaryotic and eukaryotic promoters using hidden Markov models. In ISMB-96, St. Louis, June 1996.

[135] M. G. Reese and F. H. Eeckman. Time-delay neural networks for eukaryotic promoter prediction, 1997. manuscript.

[136] M. G. Reese, F. H. Eeckman, D. Kulp, and D. Haussler. Improved splice site detection in genie. Jour. Comp. Biol., 4:311-323, 1997.

[137] Y. Sakakibara, M. Brown, I. S. Mian, R. Underwood, and D. Haussler. Stochastic context-free grammars for modeling RNA. In Proceedings of the Hawaii International Conference on System Sciences, Los Alamitos, CA, 1994. IEEE Computer Society Press. 
[138] Yasubumi Sakakibara, Michael Brown, Richard Hughey, I. Saira Mian, Kimmen Sjölander, Rebecca C. Underwood, and David Haussler. Stochastic context-free grammars for tRNA modeling. NAR, 22:5112$5120,1994$.

[139] Yasubumi Sakakibara, Michael Brown, Richard Hughey, I. Saira Mian, Kimmen Sjölander, Rebecca C. Underwood, and David Haussler. Recent methods for RNA modeling using stochastic context-free grammars. In Proceedings of the Asilomar Conference on Combinatorial Pattern Matching, New York, NY, 1994. Springer-Verlag.

[140] M. Schena, D. Shalon, R. Davis, and P. Brown. Quantitative monitoring of gene expression patterns with a complementary DNA microarray. Science, 270:467-470, October 1995.

[141] C. Scholkopf, J.C. Burges, and A.J. Smola. Advances in kernel methods : support vector learning. MIT Press, 1999.

[142] K. Sjölander, K. Karplus, M. P. Brown, R. Hughey, A. Krogh, I. S. Mian, and D. Haussler. Dirichlet mixtures: A method for improving detection of weak but significant protein sequence homology. CABIOS, 12(4):327-345, August 1996.

[143] A. Smit and P. Green. Repeatmasker2. http://ftp.genome.washington.edu/cgi-bin/RepeatMasker, 1997.

[144] P. Smyth, D. Heckerman, and M. Jordan. Probabilistic independence networks for hidden markov probability models. Neural Computation, 9:227-269, February 1997.

[145] E. Snyder and G. Stormo. Indentification of protein coding regions in genomic DNA. JMB, 248:1-18, 1995.

[146] E.L.L Sonnhammer, S.R. Eddy, and R. Durbin. Pfam: A comprehensive database of protein families based on seed alignments. Proteins, 28:405-420, 1997.

[147] M. Spingola, L. Grate, D. Haussler, and M. Ares. Genome-wide bioinformatic and molecular analysis of introns in saccharomyces cerevisiae. RNA, 5:1-13, 1999.

[148] S. Stamm, M. Q. Zhang, T. G. Marr, and D. M. Helfman. A sequence compilation and comparison of exons that are alternatively spliced in neurons. NAR, 22(9):1515-1526, 1994.

[149] G. D. Stormo and D. Haussler. Optimally parsing a sequence into different classes based on multiple types of information. In ISMB-94, Menlo Park, CA, August 1994. AAAI/MIT Press.

[150] C. M. Stultz, J. V. White, and T. F. Smith. Structural analysis based on state-space modeling. Protein Sci., 2:305-315, 1993.

[151] Christoper Tarnas and Richard Hughey. Reduced space hidden Markov model training. Bioinformatics, 14(5):401-406, 1998.

[152] Rebecca C. Underwood. Stochastic context-free grammars for modeling three spliceosomal small nuclear ribonucleic acids. Master's thesis, University of California, Computer Science, UC Santa Cruz, CA 95064, 1994.

[153] Anne Urban. Hidden Markov models in characterization, discrimination and alignment of the helicase family of proteins. Master's thesis, University of California, Computer Science, UC Santa Cruz, CA 95064, 1994.

[154] W. W. Wasserman and J. W. Fickett. Identification of regulatory regions which confer muscle-specific gene expression. Journal of Molecular Biology, 278(1):167-181, 1998.

[155] J. Weinstein et al. An information-intensive approach to the molecular pharmacology of cancer. Science, 275:343-349, January 1997.

[156] E. Wingender, A. E. Kel, O. V. Kel, H. Karas, T. Heinemeyer, P. Dietze, R. Knuppel, A. G. Romaschenko, and N. A. Kolchanov. TRANSFAC, TRRD, and COMPEL: towards a federated database system on transcriptional regulation. NAR, 25(1):265-268, 1997.

[157] C.R. Woese and G.E. Fox. Phylogenetic structure of the prokaryotic domain: the primary kingdoms. Proc. Natl. Acad. Sci. USA., 74:5088-5090, 1997.

[158] T. Wolfsberg and D. Landesman. A comparison of expressed sequence tags (ests) to human genomic sequences. Nucleic Acids Research, 25(8):1626-1632, 1997.

[159] T.D. Wu. A segment-based dynamic programing algorithm for predicting genes. Jour. Comp. Biol., $3: 375-394,1996$.

[160] R. Xu, J. Teng, and T. A. Cooper. The cardiac troponin t alternative exon contains a novel purine-rich positive splicing element. Molecular and Cellular Biology, 13:3660-3674,-1993. 
[161] Y. Xu, R. Mural, and E. Uberbacher. Inferring gene structures in genomic sequences using pattern recognition and expressed sequence tags. In Proceedings, 5th International Conference on Intelligent Systems for Molecular Biology, pages 344-353, 1997.

[162] Y. Xu and E. C. Uberbacher. Automated gene identification in large-scale genomic sequences. Journal of Computational Biology, 4(3):325-338, 1997.

[163] M. Q. Zhang. Identification of human gene core promoters in silico. Genome Research, 8(3):319 326, 1998. 


\title{
7 Biographical Sketches
}

\subsection{David Haussler}

\author{
David Haussler \\ Professor of Computer Science \\ University of California, Santa Cruz, CA 95064
}

\section{Education, Professional Appointments, Honors}

1975 Mathematics B.A., Connecticut College, Magna Cum Laude, Phi Beta Kappa Received Julia Bower Mathematics Award

1978 Applied Mathematics, M.S., Cal Poly, Mathematics Award

1982 Computer Science, Ph.D., U. Colorado, Graduate Student Research Award

1982-86 Asst Prof, Dept of Mathematics \& Computer Science, Univ of Denver, CO.

1986-89 Asst Prof, Dept of Computer Science, Univ of Calif, Santa Cruz, CA.

1989-92 Assoc Prof, Dept of Computer Science, Univ of Calif, Santa Cruz, CA.

1992- Professor, Dept of Computer Science, Univ of Calif, Santa Cruz, CA.

1992 Elected as a Fellow of the American Association for Artificial Intelligence.

\section{Professional Activities}

Associate Editor: Journal of Computational Biology

Associate Editor: Machine Learning

Editorial Board: J. Artificial Intelligence Research, J. Neurocomputing

Chairman, Steering Committee for COLT (Computational Learning Theory),1989-1992

Member: Working Group on Machine Learning, Int'l Federation for Information Processing

Member: UC System-wide Life Science Informatics Working Group

Co-organizer: Program in Neural Networks and Machine Learning, Fall 1997, and Workshop on Statistical Analysis of DNA and Protein Sequences, Oct. '97, Isaac Newton Institute for Mathematical Sciences, Cambridge, England

Panel member: DOE Human Genome Project, Informatics Section 9/93 and 9/96, NIH 11/98

Conference Chair or program committee member: 1st, 2nd, and 5th ACM Conf. on Computational Learning Theory, ISMB '94-'99, RECOMB '97 and '98, NIPS, FOCS, and other meetings

\section{Selected Papers in Biosequence Analysis}

M. Spingola, L. Grate, D. Haussler, and M. Ares (1999), "Genome-wide bioinformatic and molecular analysis of introns in Saccharomyces cerevisiae", RNA, 5, pp. 1-13.

B. Lazareva and D. Haussler (1999), A Probabilistic Approach to Consensus Multiple Alignment, Proc. Pacific Symposium on Biocomputing, pp. 150-161.

Haussler, D. (1998), "Computational Genefinding", in Trends in Biochemical Sciences, Supplementary Guide to Bioinformatics, pp. 12-15.

J. Park, K. Karplus, C. Barrett, R. Hughey, D. Haussler, T. Hubbard, and C. Chothia (1998), "Sequence comparisons using multiple sequences detect three times as many remote homologues as pairwise methods," Journal of Molecular Biology, 284(4) pp. 1201-1210.

Karplus, K., K. Sjolander, C. Barrett, M. Cline, D. Haussler, R. Hughey, L. Holm, and C. Sander (1998), "Predicting protein structure using hidden Markov models," Proteins: Structure, Function and Genetics, Supp. 1(1) pp. 134-139.

Kulp, D., Reese, M., Eeckman, F. and Haussler, D.. (1997), "Integrating Database Homology in a Probabilistic Gene Structure Model," Pacific Symposium on Biocomputing, Hawaii, World Scientific Press.

Reese, M., Kulp, D., Eeckman, F. and Haussler, D. (1997), "Improved splice site detection in Genie," Journal of Computational Biology, 4(3). 
U. Fayyad, D. Haussler and P. Stolorz (1996), "Mining Scientific Data," Comm. ACM, Vol. 39, No. 11, pp. 1501-1531.

U. Fayyad, D. Haussler and P. Stolorz (1996), "KDD for Science Data Analysis: Issues and Examples," Proc. Third International Conf. on Knowledge Discovery and Data Mining Portland.

Kulp, D., Reese, M., Eeckman, F. and Haussler, D.. (1996), "A Generalized Hidden Markov Model for the Recognition of Human Genes in DNA," Proc. of Fourth International Conference on Intelligent Systems in Molecular Biology.

Sjölander, K., Brown, M., Hughey, R., Karplus, K., Krogh, A., and Mian, I. S., Haussler, D. (1996), "Dirichlet Mixtures: A Method for Improving Detection of Weak but Significant Protein Sequence Homology," CABIOS Vol. 12, No. 4, pp. 327-345.

Gulko, B. and Haussler. D. (1996,) "Using Multiple Alignments and Phylogenetic Trees to Detect RNA Secondary Structure," Proceedings of the Pacific Symposium on Biocomputing, pp. 350-367.

Krogh, A., Brown, M., Mian, S., Sjolander, K. and Haussler, D. (1994), "Hidden Markov Models in Computational Biology: Applications to Protein Modeling," Journal of Molecular Biology 235:1501-1531.

Sakakibara, Y., Brown, M., Hughey, R., Mian, S., Sjölander, K., Underwood, R. and Haussler, D. (1994), "Stochastic Context-Free Grammars for tRNA modeling", Nucleic Acids Research Vol. 22, No. 23, pp. 51125120 .

Krogh, A., Mian, S., and Haussler, D. (1994), “A Hidden Markov Model that Finds Genes in E. coli.," Nucleic Acids Research Vol. 22, No. 22, pp. 4769-4778.

L. Grate, M. Herbster, R. Hughey, I.S. Mian, H. Noller, and D. Haussler (1994), "RNA Modeling Using Gibbs Sampling and Stochastic Context Free Grammars," Proc. of ISMB94.

G. D. Stormo and D. Haussler (1994), "Optimally Parsing a Sequence into Different Classes Based on Multiple Types of Information," Proc. of ISMB94.

Brown, M.P., Hughey, R., Krogh, A., Mian, I.S., Sjölander, K., Haussler, D. (1993), "Using Dirichlet mixture priors to derive hidden Markov models for protein families," Proc. First International Conference on Intelligent Systems for Molecular Biology, Washington D.C., July, 1993.

Milosavljevic, A., Jurka, J. and Haussler, D. (1989), "Informed parsimonious inference of prototypical genetic sequences," Proc. Second Workshop on Computational Learning Theory, Santa Cruz, CA.

Clift, B., Haussler, D., McConnell, R., Schneider, T.D., and Stormo, G. (1986) "Sequence landscapes," Nucleic Acids Research, 14 (1), 141-158.

\section{Other Recent Publications (out of approx. 100 total publications since 1980)}

1. "Mutual Information, Metric Entropy, and Risk in Estimation of Probability Distributions," Annals of Statistics, 25 (6) (Dec. 1997). (with M. Opper)

2. "A general Minimax Result for Relative Entropy," IEEE Transactions on Info. Th., Vol. 43. No. 4, (1997) pp. 1276-1280.

3. "How to use expert advice," J. ACM. Vol. 44 No. 3 (1997) pp. 427-485. (with Cesa-Bianchi, N., Y. Freund, D. Helmbold, R. Schapire, and M. Warmuth)

4. "Characterizations of Learnability for Classes of $\{0, \ldots, n\}$-valued functions," J. Computer and Systems Sci. Vol. 50, No. 1, (1995) pp. 74-86. (with S. Ben-David, N. Cesa-Bianchi and P. Long)

5. "A Generalization of Sauer's Lemma," J. Comb. Theory (A), Vol. 71, No 2, (1995) pp. 219-240. (with Phil Long)

6. "Bounds for Predictive Errors in the Statistical Mechanics of Supervised Learning," Physical Review Letters Vol. 75, No. 20, (1995) pp. 3772-3775. (with Manfred Opper)

7. "Bounds on the Sample Complexity of Bayesian Learning Using Information Theory and the VC Dimension," Machine Learning, 14 (1), (1994) 83-114. (with M. Kearns and R. Schapire) 


\title{
7.2 Kevin Karplus
}

\author{
Associate Professor, Computer Engineering \\ University of California, Santa Cruz \\ Santa Cruz, CA 95064 \\ $1-831-459-4250$ \\ karplus@cse.ucsc.edu
}

\section{Education}

Ph. D. 1983 Computer Science Stanford University

M. S. 1976 Mathematics Stanford University

B. S. 1974 Mathematics Michigan State University

\section{Research Interests}

Modeling proteins, RNA, and DNA using Markov models, hidden Markov models, stochastic context-free grammars, and other statistical techniques. Mixtures of Dirichlet distributions for estimating amino-acid frequencies. Protein structure prediction and gene-finding in DNA. Multi-purpose high-speed hardware for biosequence analysis.

\section{Research Summary}

In 1993 I switched my research focus from logic minimization to biosequence analysis. I have worked extensively with hidden Markov models and Dirichlet mixtures, and to a lesser extent with neural networks. In summer 1998, I lead a team of researchers doing protein structure prediction for the CASP3 contest.

Richard Hughey and I have built a high-speed single-board computer (Kestrel) that can accelerate the dynamic programming for sequence alignment and hidden Markov model training. I have been developing other algorithms for Kestrel that can be used in protein structure prediction. For this project, we have designed and fabricated full-custom cMOS chips in order to get 512 simple processors on a board.

\section{Selected Publications and Patents}

[1] Kevin Karplus, Christian Barrett, Melissa Cline, Mark Diekhans, Leslie Grate, and Richard Hughey. Predicting protein structure using only sequence information. Proteins: Structure, Function, and Genetics, to appear, 1999.

[2] Lawrence A. Kelley, Robert M. MacCallum, Michael Sternberg, Kevin Karplus, Daniel Fischer, Arne Elofsson, Adam Godzik, Leszek Rychlewski, Krzysztof Pawłowski(A), David Jones, Kevin Bryson, and Burkhard Rost. CAFASP-1: Critical assessment of fully automated structure prediction methods. Proteins: Structure, Function, and Genetics, to appear, 1999.

[3] Kevin Karplus, Christian Barrett, and Richard Hughey. Hidden markov models for detecting remote protein homologies. Bioinformatics, 14(10):846-856, 1998.

[4] J. Park, K. Karplus, C. Barrett, R. Hughey, D. Haussler, T. Hubbard, and C. Chothia. Sequence comparisons using multiple sequences detect twice as many remote homologues as pairwise methods. Journal of Molecular Biology, 284(4):1201-1210, 1998. http://www.mrc-lmb.cam.ac.uk/genomes/jong/ assess_paper/assess_paperNov.html.

[5] Melissa Cline and Kevin Karplus. On alignment shift and its measures. Technical Report UCSC-CRL97-27, University of California, Santa Cruz, Jack Baskin School of Engineering, UC Santa Cruz, CA 95064, February 1998.

[6] J. D. Hirschberg, D. Dahle, K. Karplus, D. Speck, and R. Hughey. Kestrel: A programmable array for sequence analysis. Journal of VLSI Signal Processing, 19:115-126, 1998.

[7] David M. Dahle, Jeffrey D. Hirschberg, Kevin Karplus, Hansjörg Keller, Eric Rice, Don Speck, Douglas H. Williams, and Richard Hughey. Kestrel: Design of an 8-bit SIMD parallel processor. In Proc. 17th Conf. on Advanced Research in VLSI, pages 145-162. Institute of Electrical and Electronic Engineers Computer Society, September 1997.

[8] Christian Barrett, Richard Hughey, and Kevin Karplus. Scoring hidden Markov models. CABIOS, 13(2):191-199, 1997.

[9] Jeffrey D. Hirschberg, Richard Hughey, Kevin Karplus, and Don Speck. Kestrel: A programmable array for sequence analysis. In Proceedings of the International Conference on Application-Specific Array Processors, pages 25-34, Los Alamitos, CA, July 1996. Institute of Electrical and Electronic Engineers Computer Society. 
[10] Kevin Karplus, Kimmen Sjölander, Christian Barrett, Melissa Cline, David Haussler, Richard Hughey, Liisa Holm, and Chris Sander. Predicting protein structure using hidden Markov models. Proteins: Structure, Function, and Genetics, Suppl. 1:134-139, 1997.

[11] K. Sjölander, K. Karplus, M. P. Brown, R. Hughey, A. Krogh, I. S. Mian, and D. Haussler. Dirichlet mixtures: A method for improving detection of weak but significant protein sequence homology. Computer Applications in the Biosciences, 12(4):327-345, August 1996.

[12] Philipp Bucher, Kevin Karplus, Nicolas Moeri, and Kay Hoffman. A flexible motif search technique based on generalized profiles. Computers and Chemistry, 20(1):3-24, January 1996.

[13] Kevin Karplus. Regularizers for estimating distributions of amino acids from small samples. In Proceedings, 3rd International Conference on Intelligent Systems for Molecular Biology, pages 188-196, Cambridge, England, July 1995.

[14] Søren Søe and Kevin Karplus. Iterating variable ordering heuristics to compute small ordered binary decision diagrams. In International Workshop on Logic Synthesis, Lake Tahoe, CA, 23-26 May 1993.

[15] Kevin Karplus. A semi-systolic architecture for decoding error-correcting codes. United States Patent 5,157,671, 20 October 1992. Rights owned by Space Systems/Loral, application filed 29 May 1990.

[16] Kevin Karplus. ITEM: an if-then-else minimizer for logic synthesis. In EuroASIC92, pages 2-7, Paris, 1-5 June 1992.

[17] Søren Søe and Kevin Karplus. Logic minimization using two-column rectangle replacement. In $A C M$ IEEE $28^{\text {th }}$ Design Automation Conference Proceedings, pages 470-473, San Francisco, CA, 17-21 June 1991.

[18] Kevin Karplus. Xmap: a technology mapper for table-lookup field-programmable gate arrays. In $A C M$ IEEE $28^{\text {th }}$ Design Automation Conference Proceedings, pages 240-243, San Francisco, CA, 17-21 June 1991.

[19] Kevin Karplus. Amap: a technology mapper for selector-based field-programmable gate arrays. In ACM IEEE $28^{\text {th }}$ Design Automation Conference Proceedings, pages 244-247, San Francisco, CA, 17-21 June 1991.

[20] Kevin Karplus and Habib Krit. A semi-systolic decoder for the PDSC-73 error-correcting code. Discrete Applied Moth, 33(1-3):109-28, 7 Nov 1991.

[21] Pak K. Chan and Kevin Karplus. Computing signal delay in general RC networks by tree/link partitioning. IEEE Transactions on Computer-Aided Design of Integrated Circuits and Systems, 9(8):898-902, August 1990.

[22] Kevin Karplus. Using if-then-else DAGs for multi-level logic minimization. In Charles L. Seitz, editor, Advanced Research in VLSI: Proceedings of the Decennial Caltech Conference on VLSI, pages 101-118, Pasadena, CA, 20-22 March 1989.

[23] Alexander R. Strong and Kevin Karplus. Wavetable-modification instrument and method for generating musical sound. United States Patent 4,649,783, 17 March 1987. Also Australian Patent 570,669, 3 March 1988 and Canadian Patent 1,215,869, 30 December 1986 and Japanese Patent 6-139341, 1996.

[24] Kevin Karplus. Exclusion constraints, a new application of graph algorithms to VLSI design. In 4 th MIT Conference on Advanced Research in VLSI, pages 123-139, Cambridge, MA, April 7-9 1986.

[25] Kevin Karplus and Gary Haggard. Finding minimal perfect hash functions. In 1986 ACM Fourteenth Annual Computer Science Conference, Cincinnati, OH, 4-6 February 1986.

[26] Alexandru Nicolau and Kevin Karplus. ROPE: a statically scheduled supercomputer architecture. In First International Conference on Supercomputing Systems, St. Petersburg, FL, December 1985.

[27] Susan Hertz, Kevin Karplus, and Jim Kadin. The Delta rule development system for speech synthesis from text. Proceedings of the IEEE, 73(11):1589-1601, November 1985.

[28] Kevin Karplus and Alex Strong. Digital synthesis of plucked-string and drum timbres. Computer Music Journal, 7(2):43-55, Summer 1983. Reprinted in The Music Machine edited by Curtis Roads.

[29] Danny Dolev, Kevin Karplus, Alan Siegel, Alex Strong, and Jeff Ullman. Optimal wiring between rectangles. In Conference Proceedings of the $13^{\text {th }}$ Annual ACM Symposium on Theory of Computing, pages 312-317, Milwaukee, WI, 11-13 May 1981.

[30] Kevin Karplus. A proof of the isomorphism of wxyz-transformals and $2 \times 2$ integer matrices under multiplication. Computers and Mathematics with Applications, 7(5):425-430, 1981. 


\title{
7.3 Richard Hughey
}

\author{
RichaRd HugheY \\ Associate Professor of Computer Engineering \\ Jack Baskin School of Engineering \\ University of California, Santa Cruz, CA 95064 \\ http://www.cse.ucsc.edu/ ${ }^{\sim} \mathrm{rph}$
}

Employment

$1997-$

1991-1997

Associate Professor, Computer Engineering, University of California, Santa Cruz

1991

Assistant Professor, Computer Engineering, University of California, Santa Cruz

Computer Engineer, Molecular Graphics and Simulation Laboratory, Division of Computer Research and Technology, National Institutes of Health.

1987-1991 Research Assistant, Brown University, Providence, RI.

\section{Education}

Ph.D. 1991

Sc.M. 1987

Brown University, Computer Science

B.S. 1985

Brown University, Computer Science

B.A. 1985

Swarthmore College, Engineering with Distinction, Tau Beta Pi

Swarthmore College, Mathematics with Distinction, Phi Beta Kappa, Sigma Xi

\section{Selected Papers}

1. K. Karplus, C. Barrett, and R. Hughey, "Hidden Markov Models for Detecting Remote Protein Homologies," Bioinformatics, 14(10):846-856, 1998.

2. R. Karchin and R. Hughey, "Weighting Hidden Markov Models for Maximum Discrimination," Bioinformatics, 14(9):772-782, 1998.

3. J. Park, K. Karplus, C. Barrett, R. Hughey, D. Haussler, T. Hubbard, and C. Chothia, "Sequence Comparisons Using Multiple Sequences Detect Twice as many Remote Homologues as Pairwise Methods," Journal of Molecular Biology, 284(4):1201-1210, 1998.

4. C. Tarnas and R. Hughey, "Reduced Space Hidden Markov Model Training," Bioinformatics, 14(5):401$406,1998$.

5. J. D. Hirschberg, D. Dahle, K. Karplus, D. Speck, and R. Hughey, "Kestrel: A Programmable Array for Sequence Analysis," Journal of VLSI Signal Processing, 19(2):115-126, 1998.

6. K. Karplus, K. Sjölander, C. Barrett, M. Cline, D. Haussler, R. Hughey, L. Holm, and C. Sander, "Predicting Protein Structure Using Hidden Markov Models," in Proteins: Structure, Function, and Genetics, Supplement 1:134-139, 1997.

7. J. Z. Dalgaard, M. J. Moser, R. Hughey, and I. S. Mian, "Statistical Modeling, Phylogenetic Analysis and Structure Prediction of a Protein Splicing Domain Common to Inteins and Hedgehog Proteins," Journal of Computational Biology, 4(2):193-214, 1997.

8. C. Barrett, R. Hughey, and K. Karplus, "Scoring Hidden Markov Models," Computer Applications in the Biosciences, 13(2):191-199, 1997.

9. J A. Grice, R. Hughey, and D. Speck, "Reduced Space Sequence Alignment," Computer Applications in the Biosciences, 13(1):45-53, 1997.

10. R. Hughey "Parallel Sequence Comparison and Alignment," Computer Applications in the Biosciences, 12(6):473-479, 1996.

11. K. Sjölander, K. Karplus, M. P. Brown, R. Hughey, A. Krogh, I. S. Mian, and D. Haussler, "Dirichlet Mixtures: A method for improving detection of weak but significant protein sequence homology," Computer Applications in the Biosciences, 12(4):327-345, 1996.

12. R. Hughey and A. Krogh, "Hidden Markov models for sequence analysis: extension and analysis of the basic method," Computer Applications in the Biosciences 12(2):95-107, 1996.

13. Y. Sakakibara, M. Brown, R. Hughey, I. S. Mian, K. Sjölander, R. Underwood, and D. Haussler, "Stochastic Context-Free Grammars for tRNA Modeling," Nucleic Acids Research 22(23):5112-5120, 1994. 
14. R. Hughey "Concurrent error detection on programmable systolic arrays," IEEE Transactions on Computers, 42(6):752-756, 1993.

15. D. Dahle, J. Hirschberg, K. Karplus, Hj. Keller, E. Rice, D. Speck, D. Williams, and R. Hughey, "Kestrel: Design of an 8-bit Programmable Parallel Processor," 17th Conference on Advanced Research in VLSI, September 1997.

16. E. Rice and R. Hughey, "Multiprecision division on an 8-bit processor," Proceedings of the 13th IEEE Symposium on Computer Arithmetic, July 1997.

17. J. D. Hirschberg, R. Hughey, K. Karplus, and D. Speck, "Kestrel: A Programmable Array for Sequence Analysis," Proceedings of the International Conference on Application-Specific Systems, Architectures, and Processors, August 1996. (See Journal Publication 5)

18. J A. Grice., R. Hughey, and D. Speck, "Parallel sequence alignment in limited space," in Proceedings of the Third International Conference on Intelligent Systems for Molecular Biology, AAAI/MIT Press, Menlo Park, California, July 1995. (See Journal Publication 9)

19. R. Hughey "Parallel Sequence Comparison and Alignment," in Proceedings of the International Conference on Application-Specific Array Processors, IEEE Computer Society Press, July 1995. (See Journal Publication 10)

20. L. Grate, M. Herbster, R. Hughey, I. S. Mian, H. Noller, and D. Haussler, "RNA Modeling Using Gibbs Sampling and Stochastic Context Free Grammars," in Proceedings of the Second International Conference on Intelligent Systems for Molecular Biology, AAAI/MIT Press, Meno Park, California, August 1994.

21. Y. Sakakibara, M. Brown, R. Hughey, I. S. Mian, K. Sjölander, R. Underwood, and D. Haussler, "Recent methods for RNA modeling using stochastic context-free grammars," in Proceedings of the Asilomar Conference on Combinatorial Pattern Matching, Springer-Verlag, June 1994. (See Journal Publication 13)

22. J. D. Roberts, R. A. Levinson, and Hughey, R., "Issues in Parallel Hardware for Conceptual Graph Retrieval," in Proceedings of the First International Conference on Conceptual Structures, August 1993. 
8 Budget Justification

The budget includes funding for the PIs to take part in and direct the project.

The first graduate student (Christian Barrett) is an outstanding senior graduate student who has already co-authored 5 papers. He is an expert in the creation and use of hidden Markov models and HMM libraries.

The second graduate student (David Lin) has worked at both the Stanford Genome Center and Roche Biosciences, and is an expert on EST analysis and mRA gene expression data.

The third graduate student (Mark Diekhans) is a phenomenal programmer who has been working with the group for three years. He implemented parts of the SAM system, the Fisher kernel method and is currently working on the PGF system.

We include travel for the PIs and graduate students to several conferences each year, as well as funds to purchase additional disks for our two 4-processor AlphaSever machines to help keep pace with the accelerating growth of genomic data.
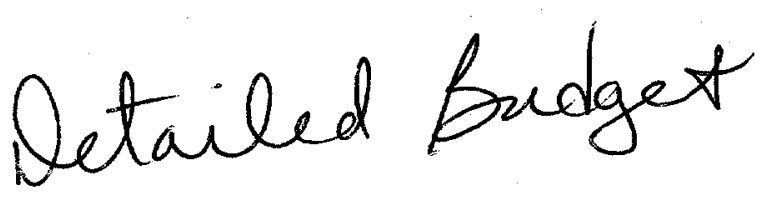

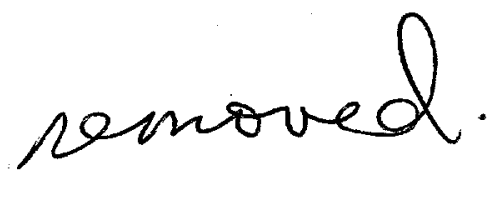

30 